\title{
Explicit filtering to obtain grid-spacing-independent and discretization-order-independent large-eddy simulation of two-phase volumetrically dilute flow with evaporation
}

\author{
Senthilkumaran Radhakrishnan ${ }^{1}$ and Josette Bellan ${ }^{1,2, \dagger}$ \\ ${ }^{1}$ Jet Propulsion Laboratory, California Institute of Technology, Pasadena, CA 91109, USA \\ ${ }^{2}$ California Institute of Technology, Pasadena, CA 91125, USA
}

(Received 16 February 2012; revised 16 October 2012; accepted 21 December 2012; first published online 19 February 2013)

Predictions from conventional large-eddy simulation (LES) are known to be gridspacing and spatial-discretization-order dependent. In a previous article (Radhakrishnan \& Bellan, J. Fluid Mech., vol. 697, 2012a, pp. 399-435), we reformulated LES for compressible single-phase flow by explicitly filtering the nonlinear terms in the governing equations so as to render the solution grid-spacing and discretization-order independent. Having shown in Radhakrishnan \& Bellan (2012a) that the reformulated LES, which we call EFLES, yields grid-spacing-independent and discretization-orderindependent solutions for compressible single-phase flow, we explore here the potential of EFLES for evaporating two-phase flow where the small scales have an additional origin compared to single-phase flow. Thus, we created a database through direct numerical simulation (DNS) that when filtered serves as a template for comparisons with both conventional LES and EFLES. Both conventional LES and EFLES are conducted with two gas-phase SGS models; the drop-field SGS model is the same in all these simulations. For EFLES, we also compared simulations performed with the same SGS model for the gas phase but two different drop-field SGS models. Moreover, to elucidate the influence of explicit filtering versus gas-phase SGS modelling, EFLES with two drop-field SGS models but no gas-phase SGS models were conducted. The results from all these simulations were compared to those from DNS and from the filtered DNS (FDNS). Similar to the single-phase flow findings, the conventional LES method yields solutions which are both grid-spacing and spatialdiscretization-order dependent. The EFLES solutions are found to be grid-spacing independent for sufficiently large filter-width to grid-spacing ratio, although for the highest discretization order this ratio is larger in the two-phase flow compared to the single-phase flow. For a sufficiently fine grid, the results are also discretization-order independent. The absence of a gas-phase SGS model leads to build-up of energy near the filter cut-off indicating that while explicit filtering removes energy above the filter width, it does not provide the correct dissipation at the scales smaller than this width. A wider viewpoint leads to the conclusion that although the minimum filter-width to grid-spacing ratio necessary to obtain the unique grid-independent solution might be 
different for various discretization-order schemes, the grid-independent solution thus obtained is also discretization-order independent.

Key words: multiphase and particle-laden flows, turbulence modelling, turbulence simulation

\section{Introduction}

Large-eddy simulation (LES) has received considerable attention over the last few decades as it currently seems to be the only methodology having the potential to compute fully turbulent flows and provide both the spatial and temporal resolution necessary for meteorological predictions, combustion process optimization and other practical applications encountered in industrial settings. In LES the governing equations are filtered and thus one solves the equations only for the large scales of the flow; the effect of the small scales is modelled by subgrid-scale (SGS) terms in the governing equations, representing the effect of the scales smaller than the filter width. It has been pointed out that SGS models are needed not because of filtering, but because of the coarser grid than that necessary to solve all flow scales (Carati, Winckelmans \& Jeanmart 2001). Despite great strides in LES, several unresolved problems remain. One of these problems plaguing model validation when comparing with a trusted template is the lack of grid independence (Pope 2004). It is often assumed that the filter width is the same as the local grid spacing, an assumption which introduces considerable error in regions where the grid spacing varies drastically, as for instance in simulations where adaptive grid refinement is used in some regions to better capture the physics (Vanella, Piomelli \& Balaras 2008). A detailed discussion of the conceptual problems raised by the lack of grid independence in LES appears in Radhakrishnan \& Bellan (2012a). In particular, it is pointed out that the issue of model accuracy cannot be addressed unless that of grid-spacing independence and discretization-order independence is solved.

Radhakrishnan \& Bellan (2012a) reformulated LES for compressible single-phase flow by explicitly filtering the nonlinear terms in the LES differential equations and also explicitly filtering the equation of state which is also nonlinear. The success of explicit filtering in rendering the results both grid-spacing and discretization-order independent was attributed to the removal of scales smaller than the filter width that would have been produced by the nonlinear terms. Performing explicit filtering also had the advantage to mandate specification of the filter shape, whereas in the conventional LES formulation the filter shape is implicit and it is only the filter width which explicitly appears. Specifying the shape of the filter is essential for validating simulations with either experimental or numerical data since these data must be themselves filtered to match the simulations. Results from filtering data using different mathematical filters, even if they have the same width, can be vastly different, and then the target of the simulations depends on the filter shape, which constitutes a problem with conventional LES. When comparing the performance of the conventional LES to the explicitly filtered LES (EFLES) for several SGS models, several conclusions were obtained by Radhakrishnan \& Bellan (2012a). (a) The conventional LES results differed according to the grid spacing and the order of spatial discretization. (b) Finer grids in conventional LES did not necessarily guarantee more favourable comparison with a template provided by a filtered direct numerical 
simulation (FDNS) database. (c) The EFLES results obtained with a lower-order discretization scheme were grid independent at a large filter-width to grid-spacing ratio as compared to the EFLES results using a higher-order spatial discretization. In other words, for higher spatial discretization order, it was possible to obtain in EFLES a grid-spacing-independent solution with a coarser grid than at lower discretization orders. It was also noted that for LES some SGS models provided a better fidelity performance for the viscous dissipation and the SGS dissipation than other models, whereas for EFLES the results were generally independent of the SGS model utilized. The comparison of SGS model performance in LES is though deceptive since what is assessed is an intertwining of numerical and modelling errors.

Because the small scales have an additional origin in evaporative two-phase flows compared to compressible single-phase flow, there is no reason to assume that the conclusions of Radhakrishnan \& Bellan (2012a) necessarily carry over to the present situation. It is well known (e.g. Gore \& Crowe 1989; Hardalupas, Taylor \& Whitelaw 1989) that according to the drop (or generally the particle) size with respect to the Kolmogorov length of the flow, the drops may either damp or enhance turbulence, so that the character of the flow could dramatically change due to the drops' presence. For example, Okong'o \& Bellan (2004) found that the global effect of the drops smaller than the Kolmogorov scale on the flow was to attenuate turbulence. For evaporating drops, the overwhelming entropy production was found for transitional flows to be due to the energy contribution of the drops to the flow, mostly through the thermodynamic phase change (Okong'o \& Bellan 2004). Moreover, even the large scales of the flow are affected by the drops' presence since the drops represent a source of vorticity at all scales (Okong'o \& Bellan 2004). This more complex physics of two-phase flow with respect to the single-phase counterpart is described by more complicated governing equations, warranting a separate examination of the performance of EFLES compared to conventional LES in this new situation.

In $\S 2$ we briefly recall the fundamental governing equations of Okong'o \& Bellan (2004). The LES equations are succinctly presented in $\S 3$ : in $\$ 3.1 .1$ for the conventional LES (formulation of Okong'o \& Bellan 2004) and in $\$ 3.1 .2$ for the EFLES (formulation of Radhakrishnan \& Bellan 2012a). The drop representation is common to conventional LES and EFLES and it is described in §3.1.3. The SGS models for both gas and drops are concisely explained in $\S 3.2$. The configuration, initial and boundary conditions are described in $\S 4$ while the numerical method, which is different from that of Okong'o \& Bellan (2004), is presented in $\S 5$. Our investigation is in the context of temporal mixing layers, the simple configuration of which avoids the effect of complex boundary conditions. Because experimental data for comparison are only available for spatial mixing layers, the present template for LES and EFLES is a FDNS database which is created for this particular purpose. The results discussed in $\S 6$ first address the DNS database in $\S 6.1$. Then in $\S 6.3$ are presented the conventional LES solutions obtained with several SGS models, various grid resolutions and spatial discretization orders, followed in $\S 6.4$ by the EFLES solutions. The results, while qualitatively similar to our single-phase compressibleflow findings (Radhakrishnan \& Bellan 2012a), are quantitatively different due to the distinct origin of the small scales in two-phase flow compared to single-phase flow, as discussed above. We explain how large-scale vorticity production by the drops is responsible for these different results. The summary and conclusions of $\S 7$ bring perspective to this study. 


\section{Governing equations for direct numerical simulation}

The governing equations are recalled for the situation of volumetrically small loading $\left(\simeq 10^{-3}\right)$, in which case the drops can be treated as point sources of mass, momentum and energy from the gas-phase perspective (Boivin, Simonin \& Squires 1998). In such situations, it is appropriate to follow the gas phase in an Eulerian framework and the liquid drops in a Lagrangian framework. Despite the small volumetric loading, the mass loadings can be considerably larger $\left(\gtrsim 10^{-1}\right)$ because the ratio of liquid density to carrier-gas density is $O\left(10^{3}\right)$, and therefore the drops may considerably influence the flow, requiring a two-way coupling between phases. We are also considering the case where the drop size is much smaller than the Kolmogorov scale, so that laminar drop evaporation models can be used.

\subsection{Gas-phase governing equations}

As in Okong'o \& Bellan (2004), we define the vector of gas-phase conservative variables $\phi=\left\{\rho, \rho u_{i}, \rho e_{t}, \rho Y_{V}\right\}$ and denote the flow field as $\phi$, where $\rho$ is the density, $u_{i}$ is the velocity in the $x_{i}$ coordinate direction, $e_{t}$ is the total energy and $Y_{V}$ is the vapour (subscript $V$ ) mass fraction (the carrier gas, subscript $C$, mass fraction is $Y_{C}$; $\left.Y_{C}+Y_{V}=1\right)$. The gas-phase conservation equations are:

$$
\begin{gathered}
\frac{\partial \rho}{\partial t}+\frac{\partial\left(\rho u_{j}\right)}{\partial x_{j}}=S_{I}, \\
\frac{\partial\left(\rho u_{i}\right)}{\partial t}+\frac{\partial\left(\rho u_{i} u_{j}\right)}{\partial x_{j}}=-\frac{\partial p}{\partial x_{i}}+\frac{\partial \sigma_{i j}}{\partial x_{j}}+S_{I I, i}, \\
\frac{\partial\left(\rho e_{t}\right)}{\partial t}+\frac{\partial\left(\rho e_{t} u_{j}\right)}{\partial x_{j}}=-\frac{\partial\left(p u_{j}\right)}{\partial x_{j}}-\frac{\partial q_{j}}{\partial x_{j}}+\frac{\partial\left(\sigma_{i j} u_{i}\right)}{\partial x_{j}}+S_{I I I}, \\
\frac{\partial\left(\rho Y_{V}\right)}{\partial t}+\frac{\partial\left(\rho Y_{V} u_{j}\right)}{\partial x_{j}}=-\frac{\partial j_{V j}}{\partial x_{j}}+S_{I},
\end{gathered}
$$

where $S_{I}, S_{I I, i}$ and $S_{I I I}$ are source terms due to interaction of the drops with the gas. The thermodynamic variables to be computed from $\phi$ are the internal energy ( $e=e_{t}-e_{k}$, where the kinetic energy is $\left.e_{k}=u_{i} u_{i} / 2\right)$, the pressure $(p)$, the temperature (T) and the enthalpy $(h=e+p / \rho)$. We assume that the perfect gas equation of state

$$
p(\phi)=\rho R(\phi) T(\phi),
$$

holds where $R(\phi)=Y_{V} R_{V}+Y_{C} R_{C}, R_{V}=R_{u} / m_{V}, R_{C}=R_{u} / m_{C}, R_{u}$ is the universal gas constant and $m_{C}$ and $m_{V}$ are the molar masses of the carrier gas and vapour respectively, and

$$
h(\phi)=h_{V} Y_{V}+h_{C} Y_{C},
$$

where $h_{C}$ and $h_{V}$ are the enthalpies of the pure gases,

$$
h_{C}=\int C_{p, C}(T) \mathrm{d} T, \quad h_{V}=\int C_{p, V}(T) \mathrm{d} T,
$$

calculated from a specified functional form of the heat capacities at constant pressure, $C_{p, C}$ and $C_{p, V}$. For the small $T$ and $p$ range of the computations, we assume the gas to be calorically perfect, meaning that $C_{p, C}$ and $C_{p, V}$ are constant, so that

$$
h_{C}(\phi)=C_{p, C} T, \quad h_{V}(\phi)=C_{p, V} T+h_{V}^{0},
$$


where $C_{p, C}=C_{p, C}\left(T^{0}\right), C_{p, V}=C_{p, V}\left(T^{0}\right)$ and $h_{V}^{0}$ is the reference vapour enthalpy at $\left(T^{0}, p^{0}\right)$ obtained from integration or tables accounting for the enthalpy difference between the vapour and carrier gas at the reference conditions. Then (2.6) becomes

$$
h(\phi)=C_{p}(\phi) T(\phi)+h_{V}^{0} Y_{V},
$$

where the mixture heat capacity at constant $p$ is $C_{p}(\phi)=C_{p, V} Y_{V}+C_{p, C} Y_{C}$. To compute $T$, we use the internal energy

$$
e(\phi)=C_{v}(\phi) T(\phi)+h_{V}^{0} Y_{V},
$$

where $C_{v}$ is the mixture heat capacity at constant volume $\left(C_{v}=C_{p}-R\right)$.

In (2.1)-(2.4), $\sigma_{i j}$ is the viscous stress, and $S_{i j}$ is the rate of strain

$$
\begin{gathered}
\sigma_{i j}(\phi)=2 \mu\left(S_{i j}-\frac{1}{3} S_{k k} \delta_{i j}\right), \\
S_{i j}(\phi)=\frac{1}{2}\left(\frac{\partial u_{i}}{\partial x_{j}}+\frac{\partial u_{j}}{\partial x_{i}}\right),
\end{gathered}
$$

where $\mu$ is the viscosity; the vapour mass flux is computed from

$$
\begin{aligned}
j_{V j}(\phi) & =\rho Y_{V} V_{V j}(\phi) \\
& =-\rho Y_{V}\left[\frac{D}{Y_{V}} \frac{\partial Y_{V}}{\partial x_{j}}+Y_{C}\left(Y_{V}+Y_{C} \frac{m_{V}}{m_{C}}\right)\left[\frac{m_{C}}{m_{V}}-1\right] \frac{D}{p(\phi)} \frac{\partial p(\phi)}{\partial x_{j}}\right],
\end{aligned}
$$

where thermal diffusion effects have been neglected, $V_{V j}$ is the vapour diffusion velocity, and $D$ is the diffusion coefficient; the carrier-gas mass flux is

$$
j_{C j}(\phi)=\rho Y_{C} V_{C j}(\phi)=-j_{V j} ;
$$

and the heat flux is

$$
q_{j}(\phi)=-\lambda \frac{\partial T(\phi)}{\partial x_{j}}+\left(h_{V}(\phi)-h_{C}(\phi)\right) j_{V j}(\phi),
$$

where $\lambda$ is the thermal conductivity. In (2.11), (2.13) and (2.15), $\mu, D$ and $\lambda$ are assumed constant, and may be defined through the Prandtl and Schmidt numbers, $\operatorname{Pr}=\mu C_{p} / \lambda$ and $S c=\mu /(\rho D)$.

\subsection{Drop (liquid-phase) governing equations}

The equations describing the evolution of the drop field have been presented in detail by Miller \& Bellan (1999) and only their essence is highlighted here. We define $Z=\left\{X_{i}, v_{i}, T_{d}, m_{d}\right\}$ as the drop field with position $X_{i}$, velocity $v_{i}$, temperature $T_{d}$, and mass $m_{d}$. Under the assumptions stated previously, the evolution equations for the drops, in a Lagrangian frame, are:

$$
\begin{gathered}
\frac{\mathrm{d} X_{i}}{\mathrm{~d} t}(Z)=v_{i}, \\
\frac{\mathrm{d} v_{i}}{\mathrm{~d} t}\left(\psi_{f}, Z\right)=\frac{1}{m_{d}} F_{i}\left(\psi_{f}, Z\right), \\
\frac{\mathrm{d} T_{d}}{\mathrm{~d} t}\left(\psi_{f}, \psi_{s}, Z\right)=\frac{1}{m_{d} C_{L}}\left[\mathscr{Q}\left(\psi_{f}, Z\right)+\dot{m}_{d}\left(\psi_{f}, \psi_{s}, Z\right) L_{V}(Z)\right], \\
\frac{\mathrm{d} m_{d}}{\mathrm{~d} t}\left(\psi_{f}, \psi_{s}, Z\right)=\dot{m}_{d}\left(\psi_{f}, \psi_{s}, Z\right),
\end{gathered}
$$


where $F_{i}$ is the drag force, $\mathscr{Q}$ is the heat flux, $\dot{m}_{d}$ is the evaporation rate, and $C_{L}$ is the heat capacity of the drop liquid. $L_{V}$ is the latent heat of vaporization, which, for calorically perfect gases, is a linear function of temperature, $L_{V}=h_{V}^{0}-\left(C_{L}-C_{p, V}\right) T_{d}$. The drop evolution depends on the gas-phase primitive variables, $\psi(\phi)=\left\{u_{i}, T, Y_{V}, p\right\}$, evaluated either at the drop surface (subscript $s$ ) or at the drop far field (subscript $f$ ). The far-field variables are taken as the gas-phase primitive variables interpolated to the drop locations. The expressions for $F_{i}, \mathscr{Q}$, and $\dot{m}_{d}$ are based on validated correlations involving point drops and Stokes drag, with the particle time constant defined by Crowe, Chung \& Troutt (1998) as $\tau_{d}=\rho_{L} d^{2} /(18 \mu)$, where $\rho_{L}$ is the density of the liquid and $d$ is the drop diameter $\left(m_{d}=\rho_{L} \pi d^{3} / 6\right)$ :

$$
\begin{gathered}
F_{i}\left(\psi_{f}, Z\right)=\frac{m_{d}}{\tau_{d}} f_{1}\left(u_{i, f}-v_{i}\right), \\
\mathscr{Q}\left(\psi_{f}, Z\right)=\frac{m_{d}}{\tau_{d}} \frac{N u}{3 P r} C_{p, f} f_{2}\left(T_{f}-T_{d}\right), \\
\dot{m}_{d}\left(\psi_{f}, Z\right)=-\frac{m_{d}}{\tau_{d}} \frac{S h}{3 S c} \ln \left[1+B_{M}\right] .
\end{gathered}
$$

In (2.20), $f_{1}$ is an empirical correlation to correct the Stokes drag for finite drop Reynolds numbers

$$
\begin{gathered}
f_{1}=\frac{1+0.0545 R e_{s l}+0.1 R e_{s l}^{1 / 2}\left(1-0.03 R e_{s l}\right)}{1+a\left|R e_{b}\right|^{b}}, \\
a=0.09+0.077 \exp \left(-0.4 R e_{s l}\right), \quad b=0.4+0.77 \exp \left(-0.04 R e_{s l}\right),
\end{gathered}
$$

based on the slip Reynolds number $R e_{s l}=\left|u_{i, f}-v_{i}\right| \rho d / \mu$, where $u_{i, f}-v_{i}$ is the slip velocity, and on the blowing Reynolds number $\operatorname{Re}_{b}=U_{b} \rho d / \mu$, where $U_{b}$ is the blowing velocity obtained from the mass conservation relation at the drop surface, $\dot{m}_{d}=-\pi \rho d^{2} U_{b}$. The correlation of (2.23) is valid for the ranges $0 \leqslant R e_{s l} \leqslant 100$ and $0 \leqslant R e_{b} \leqslant 10$. In (2.21), $C_{p, f}=C_{p, V} Y_{V, f}+C_{p, C} Y_{C, f}$ and $f_{2}=\beta /\left(\mathrm{e}^{\beta}-1\right)$, where $\beta=-1.5 \operatorname{Pr}_{d} \tau_{d} / m_{d}$ is constant for drops obeying the classical ' $d^{2}$ law' (Williams 1965). In (2.22), the mass transfer number is $B_{M}=\left(Y_{V, s}-Y_{V, f}\right) /\left(1-Y_{V, s}\right)$ where $Y_{V, s}$ is calculated directly from the surface vapour mole fraction, $X_{V, s}$, which is obtained by equating the vapour and liquid fugacities at the surface (i.e. $X_{V, s} p_{s}=p_{s a t}$; also $p_{s}=p_{f}$ ), where the saturation pressure, $p_{s a t}$, is provided by the Clausius-Clapeyron relation. Therefore,

$$
X_{V, s}=\frac{p_{a t m}}{p_{s}} \exp \left[\frac{L_{V}}{R_{V}}\left(\frac{1}{T_{B, L}}-\frac{1}{T_{d}}\right)\right], \quad Y_{V, s}=\frac{X_{V, s}}{X_{V, s}+\left(1-X_{V, s}\right) m_{C} / m_{V}},
$$

where $p_{a t m}=1$ atm and $T_{B, L}$ is the liquid saturation temperature at $p_{\text {atm }}$ (i.e. the normal boiling temperature). Finally, the Nusselt, $N u$, and Sherwood, $S h$, numbers appearing in (2.21) and (2.22) are empirically modified for convective corrections to heat and mass transfer based on the Ranz-Marshall correlations

$$
N u=2+0.552 \operatorname{Re}_{s l}^{1 / 2}(\operatorname{Pr})^{1 / 3}, \quad S h=2+0.552 \operatorname{Re}_{s l}^{1 / 2}(S c)^{1 / 3} .
$$

Except for $\tau_{d}$, which depends on $\mu$, (2.20)-(2.22) depend essentially on ratios of transport properties through non-dimensional numbers. Therefore, the value of $\tau_{d}$ and thus for a given liquid and drop size, the value of $\mu$ determines the interaction time between drops and gas. 


\subsection{Source terms in the gas-phase governing equations}

Each drop acts as a point source of mass, momentum and energy for the gas phase, with the drop source vector

$$
\begin{gathered}
S_{d}\left(\psi_{f}, Z\right)=\left\{S_{I, d}, S_{I I, i, d}, S_{I I I, d}, S_{I, d}\right\}, \\
S_{I, d}=-\dot{m}_{d}, \\
S_{I I, i, d}=-\left[F_{i}+\dot{m}_{d} v_{i}\right], \\
S_{I I I, d}=-\left[F_{i} v_{i}+\mathscr{Q}+\dot{m}_{d}\left(\frac{1}{2} v_{i} v_{i}+h_{V, s}\right)\right],
\end{gathered}
$$

where $h_{V, s}=C_{p, V} T_{d}+h_{V}^{0}$ is the vapour enthalpy at the drop surface. The drop sources in the Lagrangian frame are then reconstructed in the Eulerian frame to compute the gas-phase source vector $S$ of (2.1)-(2.4), $S\left(\psi_{f}, Z\right)=\left\{S_{I}, S_{I I, i}, S_{I I I}, S_{I}\right\}$. Following Miller \& Bellan (1999), we calculate

$$
S\left(\psi_{f}, Z\right)=\sum_{\alpha} \frac{w_{\alpha}}{V}\left[S_{d}\left(\psi_{f}, Z\right)\right]_{\alpha}
$$

where the summation is over all physical drops $\alpha$ residing within a local numerical discretization volume, $V$, and the geometrical weighting factor, $w_{\alpha}$, distributes the individual drop contributions to the eight nearest surrounding grid nodes (i.e. corners of the computational volume $V$ ) in proportion to the drop distance from those nodes.

\section{Large-eddy-simulation formulations}

To obtain the LES equations solving for the large flow-field scales, a filtering operation is applied to the governing equations. The filtering operation is defined as

$$
\bar{\psi}(\boldsymbol{x})=\int_{V_{f}} \psi(\boldsymbol{y}) G(\boldsymbol{x}-\boldsymbol{y}) \mathrm{d} \boldsymbol{y}
$$

where $G$ is the filter function and $V$ is the filtering volume; $G$ has the property that for a spatially invariant function, the filtered function is identical to the unfiltered one. For compressible flows, Favre filtering is used, which is defined as $\tilde{\psi}=\overline{\rho \psi} / \bar{\rho}$ where $\rho$ is the density.

We adopt here the same transport properties as employed in DNS, and thus in the following we only discuss the formulation of the governing equations.

\subsection{Governing equations}

\subsubsection{Conventional LES governing equations}

The filtered gas-phase equations are

$$
\begin{gathered}
\frac{\partial \bar{\rho}}{\partial t}+\frac{\partial\left(\bar{\rho} \tilde{u}_{j}\right)}{\partial x_{j}}=\bar{S}_{I}, \\
\frac{\partial\left(\bar{\rho} \tilde{u}_{i}\right)}{\partial t}+\frac{\partial\left(\bar{\rho} \tilde{u}_{i} \tilde{u}_{j}\right)}{\partial x_{j}}=-\frac{\partial[p(\bar{\phi})]}{\partial x_{i}}+\frac{\partial \sigma_{i j}(\bar{\phi})}{\partial x_{j}}+\bar{S}_{I I, i}-\frac{\partial \tau_{i j}}{\partial x_{j}}, \\
\frac{\partial\left(\bar{\rho} \tilde{e}_{t}\right)}{\partial t}+\frac{\partial\left(\bar{\rho} \tilde{e}_{t} \tilde{u}_{j}\right)}{\partial x_{j}}=-\frac{\partial\left[p(\bar{\phi}) \tilde{u}_{j}\right]}{\partial x_{j}}-\frac{\partial q_{j}(\bar{\phi})}{\partial x_{j}}+\frac{\partial\left[\sigma_{i j}(\bar{\phi}) \tilde{u}_{i}\right]}{\partial x_{j}} \\
+\bar{S}_{I I I}-\frac{\partial \zeta_{j}}{\partial x_{j}}-\frac{\partial}{\partial x_{j}}\left(\tau_{i j} \tilde{u}_{i}\right), \\
\frac{\partial\left(\bar{\rho} \tilde{Y}_{V}\right)}{\partial t}+\frac{\partial\left(\bar{\rho} \tilde{Y}_{V} \tilde{u}_{j}\right)}{\partial x_{j}}=-\frac{\partial j_{V j}(\bar{\phi})}{\partial x_{j}}+\bar{S}_{I}-\frac{\partial \eta_{j}}{\partial x_{j}}
\end{gathered}
$$


In the above equations (3.2)-(3.5), it is assumed that $\overline{f(\phi)}$ can be replaced by $f(\bar{\phi})$ for terms such as pressure, viscous stresses and heat conduction, and that the error due to this assumption is negligible (see Okong'o \& Bellan 2004). Quantities $\tau_{i j}, \zeta_{j}$ and $\eta_{j}$ in (3.2)-(3.5) are the SGS terms that represent the effect of the unresolved component on the resolved component of the flow field

$$
\tau_{i j}=\overline{\rho u_{i} u_{j}}-\bar{\rho} \tilde{u}_{i} \tilde{u}_{j}, \quad \zeta_{j}=\overline{\rho h u_{j}}-\bar{\rho} \tilde{h} \tilde{u}_{j}, \quad \eta_{j}=\overline{\rho Y_{V} u_{j}}-\bar{\rho} \tilde{Y}_{V} \tilde{u}_{j},
$$

where $\tilde{h}=\tilde{e}+\bar{p} / \bar{\rho}$.

In the conventional LES, the equation of state is

$$
p(\bar{\phi})=\bar{\rho} R(\bar{\phi}) T(\bar{\phi}) \text {. }
$$

\subsubsection{Explicitly filtered LES (EFLES) governing equations}

The conventional LES formulation is devoid of a filter shape; the only effect of filtering is through the relationship between filter width and grid spacing, making the filter implicit. The truncation error may also act as an implicit filter. Clearly, this lack of knowledge regarding the filter shape poses a problem when comparing LES numerical predictions with experiments since it is uncertain how the raw experimental data should be treated for comparison with simulations. To remedy this situation and to remove scales that are affected by numerical errors, one can reformulate the LES equations by introducing an explicit filter so as to impose a baseline for comparing experiments and simulations. In simulations, this explicit filter has the role of suppressing higher frequencies generated due to nonlinearities, thereby controlling the spectral content of the resolved flow field. Thus, in explicitly filtered LES, the nonlinear convective terms in the mass, momentum and energy equations, and the term describing pressure work in the energy equation, as well as in the equation of the state, are explicitly filtered out. An exception to explicit filtering of all nonlinear terms in the governing equations is the resolved-stress work term in the energy equation, the ad-hoc hypothesis being here (justified by the results in $\S 6$ ) that this term does not produce significant scales smaller than the filter width. Applying an explicit filter to the aforementioned nonlinear terms yields

$$
\begin{gathered}
\frac{\partial \bar{\rho}}{\partial t}+\frac{\partial \overline{\left(\bar{\rho} \tilde{u}_{j}\right)}}{\partial x_{j}}=\bar{S}_{I}, \\
\frac{\partial\left(\bar{\rho} \tilde{u}_{i}\right)}{\partial t}+\frac{\partial \overline{\left(\bar{\rho} \tilde{u}_{i} \tilde{u}_{j}\right)}}{\partial x_{j}}=-\frac{\partial[p(\bar{\phi})]}{\partial x_{i}}+\frac{\partial \sigma_{i j}(\bar{\phi})}{\partial x_{j}}+\bar{S}_{I I, i}-\frac{\partial \tau_{i j}^{e f}}{\partial x_{j}}, \\
\frac{\partial\left(\bar{\rho} \tilde{e}_{t}\right)}{\partial t}+\frac{\partial \overline{\left(\bar{\rho} \tilde{e}_{t} \tilde{u}_{j}\right)}}{\partial x_{j}}=-\frac{\partial \overline{\left[p(\bar{\phi}) \tilde{u}_{j}\right]}}{\partial x_{j}}-\frac{\partial q_{j}(\bar{\phi})}{\partial x_{j}}+\frac{\partial\left[\sigma_{i j}(\bar{\phi}) \tilde{u}_{i}\right]}{\partial x_{j}} \\
+\bar{S}_{I I I}-\frac{\partial \zeta_{j}^{e f}}{\partial x_{j}}-\frac{\partial}{\partial x_{j}}\left(\tau_{i j}^{e f} \tilde{u}_{i}\right), \\
\frac{\partial\left(\bar{\rho} \tilde{Y}_{V}\right)}{\partial t}+\frac{\partial \overline{\left(\bar{\rho} \tilde{Y}_{V} \tilde{u}_{j}\right)}}{\partial x_{j}}=-\frac{\partial j_{V j}(\bar{\phi})}{\partial x_{j}}+\bar{S}_{I}-\frac{\partial \eta_{j}^{e f}}{\partial x_{j}},
\end{gathered}
$$

having neglected other SGS terms the modelling of which would only add to the accuracy of the calculation but would not address the present focus which is gridspacing and discretization-order independence.

Quantities $\tau_{i j}^{e f}, \zeta_{j}^{e f}$ and $\eta_{j}^{e f}$ in (3.9)-(3.11) are the SGS terms for the explicitly filtered case (denoted by superscript ef) and are different from the SGS terms that 
appear in (3.2)-(3.5)

$$
\tau_{i j}^{e f}=\overline{\rho u_{i} u_{j}}-\overline{\bar{\rho} \tilde{u}_{i} \tilde{u}_{j}}, \quad \zeta_{j}^{e f}=\overline{\rho h u_{j}}-\overline{\bar{\rho} \tilde{h} \tilde{u}_{j}}, \quad \eta_{j}^{e f}=\overline{\rho Y_{V} u_{j}}-\overline{\bar{\rho} \tilde{Y}_{V} \tilde{u}_{j}} .
$$

Since the equation of state is nonlinear, it is filtered to obtain the pressure as

$$
\bar{p}(\phi)=\overline{\bar{\rho} \tilde{R}(\bar{\phi}) \tilde{T}(\bar{\phi})}
$$

Equations (3.14) and (3.15) (below) remain valid for computing $\bar{S}\left(\psi_{f}, Z\right)$, except that the vector $\psi_{f}$ is now based on the solution of the explicitly filtered LES equations.

\subsubsection{Filtered source terms}

From (3.1), the filtered source terms (FSTs) $\left(\bar{S}=\left\{\bar{S}_{I}, \bar{S}_{I I, i}, \bar{S}_{I I I}, \bar{S}_{I}\right\}\right)$ are properly interpreted by considering a drop located at $\boldsymbol{X}$ within the filtering volume $V_{f}$ and its contribution within that volume

$$
\bar{S}(\boldsymbol{x})=\int_{V_{f}} S_{d} \delta(\boldsymbol{y}-\boldsymbol{X}) G(\boldsymbol{x}-\boldsymbol{y}) \mathrm{d} \boldsymbol{y},
$$

where $S_{d} \delta(\boldsymbol{y}-\boldsymbol{X})$ is the point-source contribution from the drop and $\delta$ is the delta function. When $G$ is a top-hat filter, the FST is

$$
\bar{S}\left(\psi_{f}, Z\right)=\frac{1}{V_{f}} \sum_{\beta}\left[S_{d}\left(\psi_{f}, Z\right)\right]_{\beta},
$$

a volume-average over the drops $\beta$ within the filtering volume, where $S_{d}$ was defined by (2.27). Since $\bar{S}$ is not known in LES, it must be modelled by invoking $\bar{\phi}$. Similarly, if the drop field to which the $\beta$ drop belongs is different from that to which the $\alpha$ drop belongs, there will also be a drop-field model. Because the drop-field model is at a scale smaller than the LES grid, this is called a drop-field SGS model. This terminology is consistent with the $\bar{S}\left(\psi_{f}, Z\right)$ functional dependence upon both gas-field and drop-field variables.

The filtered equations contain terms that cannot be computed directly from $\bar{\phi}$ and that therefore need to be modelled, namely: $(a)$ the SGS fluxes $\left(\tau_{i j}, \zeta_{j}, \eta_{j}\right)$ or $\left(\tau_{i j}^{e f}, \zeta_{j}^{e f}, \eta_{j}^{e f}\right)$ for which models are presented in $\S 3.2$; and $(b)$ the FSTs $\left(\bar{S}_{I}, \bar{S}_{I I, i}, \bar{S}_{I I I}, \bar{S}_{I}\right)$ for which the model described below in $\S 3.2 .3$ is adopted, as being that recommended by Okong'o \& Bellan (2004).

\subsection{SGS models: gas and drops}

\subsubsection{Conventional LES SGS gas models}

Four types of models were employed for the SGS terms $\left(\tau_{i j}, \eta_{j}, \zeta_{j}\right)$ but only two of them are described here, for brevity. Additional to the two models presented below, the Gradient model (Clark, Ferziger \& Reynolds 1979; Liu, Meneveau \& Katz 1994; Okong'o \& Bellan 2004) and the Clark model (Clark et al. 1979) were also used in simulations and the results obtained were qualitatively similar to the following two models.

(a) The Smagorinsky $(1963,1993)$ model is an eddy viscosity model derived assuming that the production of the subgrid-scale turbulent kinetic energy is in balance with the dissipation of the subgrid-scale turbulent kinetic energy. In this model

$$
\tau_{i j}-\frac{1}{3} \tau_{k k} \delta_{i j}=-2 C_{S M}^{2} \bar{\Delta}^{2} \bar{\rho}|S(\bar{\phi})|\left[S_{i j}(\bar{\phi})-\frac{\delta_{i j}}{3} S_{k k}(\bar{\phi})\right],
$$


where $S^{2}(\phi)=2 S_{i j}(\phi) S_{i j}(\phi)$ and the Yoshizawa (1986) (YO) model is used to compute

$$
\tau_{k k}=C_{Y O} \bar{\Delta}^{2} S^{2}(\bar{\phi}) .
$$

The SGS scalar fluxes, vapour mass fraction or enthalpy, are modelled as

$$
\begin{gathered}
\eta_{j}=-C_{S M}^{2} \frac{\bar{\Delta}^{2} \bar{\rho}|S(\bar{\phi})|}{P r_{s g s}} \frac{\partial \widetilde{Y}_{v}}{\partial x_{j}}, \\
\zeta_{j}=-C_{S M}^{2} \frac{\bar{\Delta}^{2} \bar{\rho}|S(\bar{\phi})|}{S c_{s g s}} \frac{\partial \widetilde{h}^{2}}{\partial x_{j}} .
\end{gathered}
$$

Coefficients $C_{S M}, P r_{s g s}$, which is interpreted as an SGS Prandtl number, and $S c_{s g s}$, which is interpreted as an SGS Schmidt number, are computed through the dynamic coefficient computation described in $\S \mathrm{A} .1$ and the model name is abbreviated as LES-DSM.

(b) The mixed model combines the Smagorinsky model with a scale-similarity model which postulates similarity between the SGS and the small resolved scale (Bardina, Ferziger \& Reynolds 1980; Pruett, Sochacki \& Adams 2001). In the scalesimilarity model, the flow is additionally filtered to obtain the SGS terms:

$$
\begin{aligned}
& \tau_{i j}-\frac{\delta_{i j}}{3} \tau_{k k}=-2 C_{S M}^{2} \bar{\Delta} \bar{\rho}|S(\bar{\phi})|\left(S_{i j}(\bar{\phi})-\frac{\delta_{i j}}{3} S_{k k}(\bar{\phi})\right)+\bar{\rho}\left(\widetilde{\tilde{u}_{i} \tilde{u}_{j}}-\tilde{\tilde{u}}_{i} \tilde{\tilde{u}}_{j}\right), \\
& \tau_{k k}=C_{Y O} \bar{\Delta}^{2} S^{2}(\bar{\phi})+\bar{\rho}\left(\widetilde{\tilde{u}_{i} \tilde{u}_{i}}-\tilde{\tilde{u}}_{i} \tilde{\tilde{u}}_{i}\right), \\
& \eta_{j}=-C_{S M}^{2} \frac{\bar{\Delta}^{2} \bar{\rho}|S(\bar{\phi})|}{\operatorname{Pr}_{s g s}} \frac{\partial \widetilde{Y}_{v}}{\partial x_{j}}+\bar{\rho}\left(\widetilde{\tilde{Y}_{V} \tilde{u}_{j}}-\widetilde{\tilde{Y}_{V}} \widetilde{\tilde{u}}_{j}\right), \\
& \zeta_{j}=-C_{S M}^{2} \frac{\bar{\Delta}^{2} \bar{\rho}|S(\bar{\phi})|}{S c_{s g s}} \frac{\partial \widetilde{h}}{\partial x_{j}}+\bar{\rho}\left(\widetilde{\tilde{h}} \tilde{u}_{j}-\tilde{\tilde{h}} \widetilde{\tilde{u}}_{j}\right) .
\end{aligned}
$$

The model name is abbreviated as LES-DMM.

\subsubsection{Explicitly filtered LES SGS gas models}

Since the SGS terms in the EFLES formulation are different from the ones in the LES formulation, the SGS models for the SGS stresses are reformulated as follows:

(a) The Smagorinsky model for the SGS stresses is reformulated by applying an explicit filter to (3.16) so as to remove the scales below the filter width that are generated by the nonlinearity of the model,

$$
\tau_{i j}^{e f}-\frac{1}{3} \tau_{k k}^{e f} \delta_{i j}=-\overline{2 C_{S M}^{2} \bar{\Delta}^{2} \bar{\rho}|S(\bar{\phi})|\left[S_{i j}(\bar{\phi})-\frac{\delta_{i j}}{3} S_{k k}(\bar{\phi})\right]} .
$$

The Yoshizawa model for the trace term is

$$
\tau_{k k}^{e f}=\overline{C_{Y O} \bar{\Delta}^{2} S^{2}(\bar{\phi})} .
$$

The SGS scalar fluxes, vapour mass fraction or enthalpy, are modelled as

$$
\begin{aligned}
\eta_{j}^{e f} & =-\frac{C_{S M}^{2} \frac{\bar{\Delta}^{2} \bar{\rho}|S(\bar{\phi})|}{P r_{s g s}} \frac{\partial \widetilde{Y}_{v}}{\partial x_{j}}}{\widetilde{D}_{j}} \\
\zeta_{j}^{e f} & =-C_{S M}^{2} \frac{\bar{\Delta}^{2} \bar{\rho}|S(\bar{\phi})|}{S c_{s g s}} \frac{\partial x_{j}}{\partial x_{j}}
\end{aligned}
$$


The coefficients $C_{S M}, P r_{s g s}$ and $S c_{s g s}$ are dynamically computed as described in $\S$ A.2.

(b) The mixed model in the explicitly filtered case is obtained by combining the above Smagorinsky model and a scale-similarity term. In this case, the scale-similarity term is evaluated using the actual expression for SGS terms ((3.9)-(3.11)) with the full flow field $(\phi)$ replaced by the resolved flow field $(\bar{\phi})$

$$
\begin{aligned}
& \tau_{i j}^{e f}-\frac{\delta_{i j}}{3} \tau_{k k}^{e f}=-\overline{2 C_{S M}^{2} \bar{\Delta} \bar{\rho}|S(\bar{\phi})|\left(S_{i j}(\bar{\phi})-\frac{\delta_{i j}}{3} S_{k k}(\bar{\phi})\right)}+\left(\overline{\bar{\rho} \tilde{u}_{i} \tilde{u}_{j}}-\overline{\bar{\rho} \tilde{\tilde{u}}_{i} \widetilde{\tilde{u}}_{j}}\right), \\
& \tau_{k k}^{e f}=\overline{C_{Y O} \bar{\Delta}^{2} S^{2}(\bar{\phi})}+\left(\overline{\bar{\rho} \tilde{u}_{i} \tilde{u}_{i}}-\overline{\bar{\rho} \widetilde{\tilde{u}}_{i} \widetilde{\tilde{u}}_{i}}\right), \\
& \eta_{j}^{e f}=-\overline{C_{S M}^{2} \frac{\bar{\Delta}^{2} \bar{\rho}|S(\bar{\phi})|}{P r_{s g s}} \frac{\partial \widetilde{Y}_{v}}{\partial x_{j}}}+\left(\overline{\bar{\rho} \tilde{Y}_{V} \tilde{u}_{j}}-\overline{\bar{\rho} \widetilde{Y}_{V} \tilde{\tilde{u}}_{j}}\right), \\
& \zeta_{j}^{e f}=-\overline{C_{S M}^{2} \frac{\bar{\Delta}^{2} \bar{\rho}|S(\bar{\phi})|}{S c_{s g s}} \frac{\partial \widetilde{h}}{\partial x_{j}}}+\left(\overline{\bar{\rho} \tilde{h} \tilde{u}_{j}}-\overline{\bar{\rho} \tilde{\tilde{h}} \widetilde{\tilde{u}_{j}}}\right),
\end{aligned}
$$

The model name is abbreviated as EFLES-DMM.

\subsubsection{Filtered-source-terms model}

In LES, the effect of the drops on the gas phase occurs through the FSTs, $\bar{S}$. To compute $\bar{S}$ through (3.15), one must have models for both the unfiltered variables at the drop far field $\left(\psi_{f}\right)$ and the drop state $(Z)$. Because $\psi_{f}$ is not available in LES, it must be modelled from the simulated flow field $(\bar{\phi})$, leading to $\psi_{f, m}$. We follow here the strategy of Leboissetier, Okong'o \& Bellan (2005) in using the filtered flow field at the drop location as a model for the unfiltered flow field (i.e. $\psi_{f, m}=\tilde{\psi}_{f}$ ), meaning that we neglect direct SGS flow effects on the drop evolution. This implies that we could presumably improve the performance of LES and EFLES by reconstructing the flow field at the drop locations using, for example, the approximate deconvolution model (ADM) of Stolz \& Adams (1999) and Stolz, Adams \& Kleiser (2001). However, because our goal is to compare the LES and EFLES performance by utilizing the same $\psi_{f, m}$ model in both simulations, our study is entirely consistent and unbiased. Future improvements in the simulation accuracy could be obtained by incorporating a flow reconstruction such as ADM or by using an SGS model to more accurately render the flow field at drop locations such as the one proposed by Bini \& Jones (2008). But the subject of accuracy can only be addressed when that of grid-spacing and discretization-order independence have been solved.

Regarding $Z$, we follow the LES spirit of reducing the resolution requirements and as in Okong'o \& Bellan (2004) we simulate a reduced drop field, here denoted $\bar{Z}$, implying that $\bar{S}$ now must be modelled from $\bar{Z}$. The model of $Z$ through $\bar{Z}$ represents the drop-field SGS model. Defining the filtered gas-phase primitive variables as $\tilde{\psi}=\left\{\tilde{u}_{i}, \tilde{T}, \tilde{Y}_{V}, \bar{p}\right\}$, then consistent with the gas-phase equations, $\tilde{\psi}(\bar{\phi})$ takes the same form as $\psi(\phi)$.

Following Okong'o \& Bellan (2004) and considering that experimental measurements only provide the total number of drops, we make a 'blind' choice to represent the physical drops $(Z)$ by a computational drop field $(\bar{Z})$ in which each computational drop represents a number, $N_{R}$, of physical drops. Thus, we define

$$
N_{R} \equiv N_{\alpha} / N_{\beta}
$$




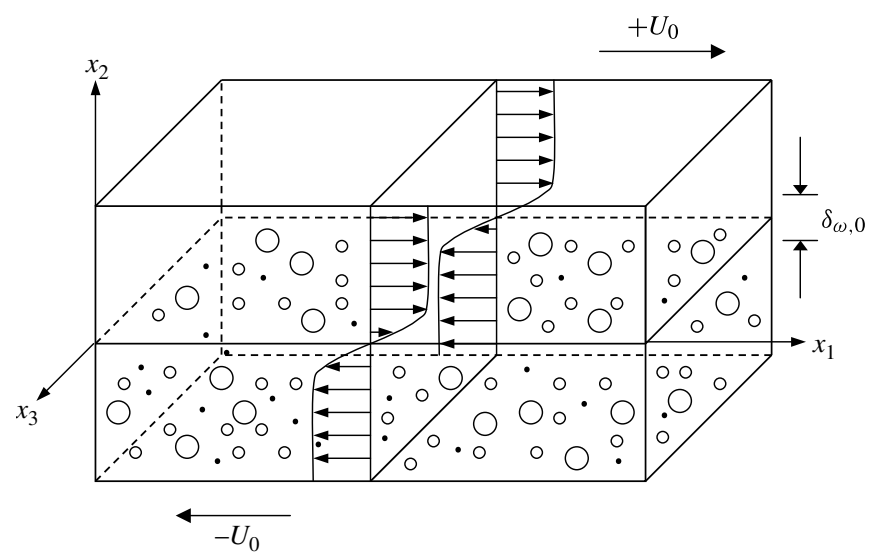

FIGURE 1. Mixing layer configuration.

as the ratio between the number of physical drops, $N_{\alpha}$, and the number of computational drops, $N_{\beta}$. The FSTs are then computed for the $N_{\beta}$ drops, and scaled by $N_{R}$ leading to

$$
\bar{S}_{m}\left(\psi_{f, m}(\bar{\phi}), \bar{Z}\right)=N_{R} \sum_{\beta} \frac{1}{V_{f}}\left[S_{d}\left(\psi_{f, m}(\bar{\phi}), \bar{Z}\left(N_{R}\right)\right)\right]_{\beta} .
$$

As in (3.15), the summation is over the drops within the filtering volume $V_{f}$, but now over a representative drop field $(\bar{Z})$ rather than the physical drops $(Z)$. The source-term contributions for each computational drop are calculated in the same manner as for physical drops, that is, the representative nature of each drop is entirely manifested in the parameter $N_{R}$. Thus, changing the value of $N_{R}$ is tantamount to changing the FST (i.e. the drop-field SGS) model.

\section{Configuration, initial and boundary conditions}

The physical configuration is that of a temporal mixing layer, shown in figure 1 , having the streamwise $\left(x_{1}\right)$, cross-stream $\left(x_{2}\right)$, and spanwise $\left(x_{3}\right)$ dimension of 0.6 , 0.45 and $0.15 \mathrm{~m}$ respectively. Periodic boundary conditions are used in the $x_{1}$ and $x_{3}$ directions, and an adiabatic slip wall condition (Poinsot \& Lele 1992) is used at the $x_{2}$ boundaries. In LES, this treatment led to instabilities at the $x_{2}$ boundaries and a sponge layer (Israeli \& Orszag 1981) near the $x_{2}$ boundaries was needed to keep the simulation stable. Drops reaching the slip walls are assumed to stick to the wall, but are otherwise transported according to the drop equations. Initially, the gas phase consists only of the carrier gas (air with no vapour); the initial mean streamwise velocity has an error-function profile. To promote transition to turbulence, the layer is initially perturbed with homogeneous noise. We add fluctuations, similar to the one used by Pantano \& Sarkar (2002), the spectrum of which is defined by

$$
E(k)=\left(\frac{k}{k_{0}}\right)^{4} \exp \left(-2\left(k / k_{0}\right)^{2}\right)
$$

where $k$ is the wavenumber and $k_{0}$ is the peak wavenumber. The turbulence intensity is $10 \%$, it is added only in the shear layer and gradually decays to zero as one moves 
away from the shear layer along the cross-stream direction. This description pertains to the DNS.

For LES or EFLES, the initial conditions are those of the filtered DNS (FDNS) at $t^{* *} \equiv t \Delta U_{0} / \delta_{\omega, 0}=100$ when the flow transitioned to turbulence. Transition to turbulence was identified from the skewness of the streamwise derivative of the streamwise component of the velocity which is -0.4 , being well within the necessary range of -0.35 to -0.5 (Lesieur 1997) for turbulent flows. $\Delta U_{0}=2 U_{0}$ is the velocity difference across the layer, $U_{0}$ is the free-stream velocity and $\delta_{\omega, 0}=\delta_{\omega}(0)$ is the initial vorticity thickness, where $\delta_{\omega}(t)=\Delta U_{0} /\left(\partial\left\langle u_{1}\right\rangle / \partial x_{2}\right)_{\max }$; the initial mean streamwise velocity has an error-function profile, as in DNS. The boundary conditions are also the same as in the DNS.

$U_{0}=M_{c, 0} a_{C, 0}$ is calculated from a specified value of the convective Mach number $M_{c, 0}$ based on the carrier-gas initial speed of sound $a_{C, 0}=\sqrt{R T_{0} C_{p} / C_{v}}$ where $T_{0}$ is the initial uniform temperature of the gas at the initial uniform (atmospheric) pressure. The specified value of the initial Reynolds number, $\operatorname{Re}_{0}=\rho_{0} \Delta U_{0} \delta_{\omega, 0} / \mu$, where $\rho_{0}$ is the initial gas density, is used to calculate $\mu$. The thermal conductivity is then computed using this value of $\mu$ and a (constant) specified value of Prandtl number, $\operatorname{Pr}=0.67$ and $S c=P r$.

Initially, $71411456 \mathrm{n}$-decane drops, corresponding to a mass loading (ratio of liquid mass to the carrier-gas mass) of 0.2 , are randomly distributed throughout the bottom half $\left(x_{2}<0\right)$ of the domain. The initial velocity of each drop is the same as that of the gas phase at its location. The drops interact only dynamically with the flow up to $t^{* *}=100$, after which the drops are allowed to evaporate and the computation is conducted up to $t^{* *}=250$. At $t^{* *}=100$, the drops are at a temperature of $345 \mathrm{~K}$ which is lower than the boiling point $(447.7 \mathrm{~K})$ of $\mathrm{n}$-decane, and also lower than the temperature of the air $(375 \mathrm{~K})$. The drop size variation is specified by a Gaussian distribution such that the initial Stokes number $\left(S t \equiv \tau_{d} \Delta U_{0} / \delta_{\omega, 0}\right)$ mean is 3 and the standard deviation is 0.5 .

\section{Numerical methodology}

The nonlinear terms in the governing equations were recast in cubic skew-symmetric form, as proposed by Kennedy \& Gruber (2008), to reduce aliasing error. DNS were performed using a fourth-order explicit Runge-Kutta scheme for temporal integration and an eighth-order central finite-difference scheme for spatial discretization; to remove aliasing errors, a sixteenth-order filter was used (Kennedy \& Carpenter 1994). Unlike in Miller \& Bellan (1999) and Okong'o \& Bellan (2004), the grid resolution is such that no smoothing of the source terms $S\left(\psi_{f}, Z\right)$ is required, as the simulation is numerically stable.

In LES or EFLES, spatial discretization was performed using the fourth-, sixthand eighth-order central schemes proposed by Kennedy \& Carpenter (1994) for the first-derivative terms in the differential equations. For the second-derivative terms in the LES or EFLES differential equations, fourth-, sixth- and eighth-order narrow stencils proposed by Mattsson \& Nordstrom (2004) were used. The narrow stencil discretization used for second-derivative terms provides better damping for the highest resolved wavenumbers, and thus made it unnecessary to apply the dealiasing filter in LES or EFLES calculations. The same Runge-Kutta temporal integration scheme utilized in DNS is used for LES and EFLES. Details of the filter applied in EFLES calculations can be obtained from Radhakrishnan \& Bellan (2012a). 


\section{Results}

We first present a description of the DNS database, which when filtered represents the template to be reached by the LES solution. Since the mixing layer evolution is self-similar, the gas-phase statistics are invariant at different $t^{* *}$ when plotted along the similarity coordinate, $\xi \equiv x_{2} / \delta_{m}(t)$ (where the momentum thickness $\delta_{m}(t)$ is defined in $\S 6.1)$. This fact has been utilized to obtain smoother gas-phase statistics than when computing them at fixed $t^{* *}$, by temporally averaging the quantities from $t^{* *}=150$ to $t^{* *}=250$, in addition to averaging along the homogeneous directions $\left(x_{1}\right.$ and $\left.x_{3}\right)$. For the drops, since their number and size vary substantially in the flow with time, we either average over a very small number of instantaneous fields or present information at a single time, $t^{* *}=250$. We first describe results obtained with the conventional LES formulation using $N_{R}=8$. Further, we discuss results from the EFLES computations, also using $N_{R}=8$ in order to compare with the LES results. We also perform EFLES with $N_{R}=16$ so as to assess the validity of the EFLES findings with a different drop-field SGS model which is less computationally intensive than for $N_{R}=8$.

\subsection{Description of the DNS database}

The DNS database consists of a single simulation initiated with $\operatorname{Re}_{0}=1200$, $M_{c, 0}=0.35, T_{C, 0}=375 \mathrm{~K}, \rho_{0}=0.9415 \mathrm{~kg} \mathrm{~m}^{-3}, \Delta U_{0}=271.7 \mathrm{~m} \mathrm{~s}^{-1}$ and $\delta_{\omega, 0}=$ $6.667 \times 10^{-3} \mathrm{~m}$. The number of grid nodes along the streamwise, the cross-stream and the spanwise directions $\left(N_{1} \times N_{2} \times N_{3}\right)$ is $1120 \times 840 \times 280$, and the ratio of the grid spacing to the Kolmogorov scale is $\Delta_{D N S} / \eta_{K}=2.5$. At $t^{* *}=0, \delta_{m, 0} / \delta_{\omega, 0}=0.22475$, where

$$
\delta_{m}=\frac{1}{\rho_{0} \Delta U_{0}^{2}} \int_{-L_{2}}^{L_{2}} \rho\left(0.5 \Delta U_{0}-\frac{\left\langle\bar{\rho} \widetilde{u}_{1}\right\rangle}{\langle\bar{\rho}\rangle}\right)\left(0.5 \Delta U_{0}+\frac{\left\langle\bar{\rho} \widetilde{u}_{1}\right\rangle}{\langle\bar{\rho}\rangle}\right) \mathrm{d} x_{2}
$$

measures the growth of the mixing layer. The layer achieves self-similarity at $t^{* *}=60$ when the growth of $\delta_{m}$ becomes linear. As already stated, the simulation is conducted up to $t^{* *}=250$ at which time station $\delta_{m} / \delta_{\omega, 0}=3.2629$ and $R e_{m} \equiv$ $\rho_{0} \Delta U_{0} \delta_{m} / \mu=3915.2$. Thus, the value of $R e_{m}$ at $t^{* *}=250$ is almost twice that achieved in the smaller Reynolds number $\left(R e_{0}=600, R e_{m}=2007.2\right)$ DNS performed in Radhakrishnan \& Bellan (2012a) but it is smaller than the $\boldsymbol{R e}_{m}=6405.1$ value achieved at the higher Reynolds number $\left(\operatorname{Re}_{0}=1800\right)$ used in Radhakrishnan \& Bellan (2012a).

\subsection{Acronyms denoting the simulations}

Considering the large number of simulations, it is useful to introduce abbreviations to label them. Since all simulations are performed with the same $R e_{0}=1200$, the distinction among simulations depends on whether it is DNS, LES, EFLES, on which gas-phase model (DSM and DMM; or NM standing for 'no model') or drop-field SGS model ( $N_{R}$ value; NR8 or NR16) is used, on the utilized grid spacing (C: coarse; M: medium; F: fine), and on the employed discretization order (fourth, sixth or eighth). Acronyms are provided in table 1 for the DNS, all LES-DSM as defined in $\$ 3.2 .1$ and all EFLES-DSM as defined in $\$ 3.2 .2$. For example, the EFLES performed with the DSM model, with a reduction of the drop field by a factor of 8 and using a medium grid spacing in conjunction with a sixth-order discretization is denoted by EFLES-DSM-M6-NR8. 


\begin{tabular}{|c|c|c|c|c|c|c|}
\hline Simulation name & $N_{R}$ & $\begin{array}{l}\text { Discretization } \\
\text { order }\end{array}$ & $\bar{\Delta}$ & $\Delta_{L E S}$ & $N_{1} \times N_{2} \times N_{3}$ & $\begin{array}{c}\text { LES/EFLES } \\
\text { grid name }\end{array}$ \\
\hline DNS & - & 8th & - & - & $1120 \times 840 \times 280$ & - \\
\hline LES-DSM-C4-NR8 & 8 & 4th & $4 \Delta_{D N S}$ & $4 \Delta_{D N S}$ & $280 \times 210 \times 70$ & $\mathrm{C}$ \\
\hline LES-DSM-C6-NR8 & 8 & 6 th & $4 \Delta_{D N S}$ & $4 \Delta_{D N S}$ & $280 \times 210 \times 70$ & $\mathrm{C}$ \\
\hline LES-DSM-C8-NR8 & 8 & 8 th & $4 \Delta_{D N S}$ & $4 \Delta_{D N S}$ & $280 \times 210 \times 70$ & $\mathrm{C}$ \\
\hline LES-DSM-M4-NR8 & 8 & 4th & $2 \Delta_{D N S}$ & $2 \Delta_{D N S}$ & $560 \times 420 \times 140$ & M \\
\hline LES-DSM-M6-NR8 & 8 & 6 th & $2 \Delta_{D N S}$ & $2 \Delta_{D N S}$ & $560 \times 420 \times 140$ & M \\
\hline LES-DSM-M8-NR8 & 8 & 8th & $2 \Delta_{D N S}$ & $2 \Delta_{D N S}$ & $560 \times 420 \times 140$ & M \\
\hline EFLES-DSM-C4-NR8 & 8 & 4th & $8 \Delta_{D N S}$ & $4 \Delta_{D N S}$ & $280 \times 210 \times 70$ & $\mathrm{C}$ \\
\hline EFLES-DSM-C4-NR16 & 16 & 4th & $8 \Delta_{D N S}$ & $4 \Delta_{D N S}$ & $280 \times 210 \times 70$ & $\mathrm{C}$ \\
\hline EFLES-DSM-C6-NR8 & 8 & 6 th & $8 \Delta_{D N S}$ & $4 \Delta_{D N S}$ & $280 \times 210 \times 70$ & $\mathrm{C}$ \\
\hline EFLES-DSM-C6-NR16 & 16 & 6 th & $8 \Delta_{D N S}$ & $4 \Delta_{D N S}$ & $280 \times 210 \times 70$ & $\mathrm{C}$ \\
\hline EFLES-DSM-C8-NR8 & 8 & 8th & $8 \Delta_{D N S}$ & $4 \Delta_{D N S}$ & $280 \times 210 \times 70$ & $\mathrm{C}$ \\
\hline EFLES-DSM-C8-NR16 & 16 & 8 th & $8 \Delta_{D N S}$ & $4 \Delta_{D N S}$ & $280 \times 210 \times 70$ & $\mathrm{C}$ \\
\hline EFLES-DSM-M4-NR8 & 8 & 4th & $8 \Delta_{D N S}$ & $2 \Delta_{D N S}$ & $560 \times 420 \times 140$ & M \\
\hline FLES-DSM-M4-NR16 & 16 & 4th & $8 \Delta_{D N S}$ & $2 \Delta_{D N S}$ & $560 \times 420 \times 140$ & M \\
\hline EFLES-DSM-M6-NR8 & 8 & 6 th & $8 \Delta_{D N S}$ & $2 \Delta_{D N S}$ & $560 \times 420 \times 140$ & M \\
\hline EFLES-DSM-M6-NR16 & 16 & 6 th & $8 \Delta_{D N S}$ & $2 \Delta_{D N S}$ & $560 \times 420 \times 140$ & M \\
\hline EFLES-DSM-M8-NR8 & 8 & 8 th & $8 \Delta_{D N S}$ & $2 \Delta_{D N S}$ & $560 \times 420 \times 140$ & M \\
\hline EFLES-DSM-M8-NR16 & 16 & 8 th & $8 \Delta_{D N S}$ & $2 \Delta_{D N S}$ & $560 \times 420 \times 140$ & M \\
\hline EFLES-DSM-F4-NR8 & 8 & 4th & $8 \Delta_{D N S}$ & $\Delta_{D N S}$ & $1120 \times 840 \times 280$ & $\mathrm{~F}$ \\
\hline EFLES-DSM-F6-NR8 & 8 & 6 th & $8 \Delta_{D N S}$ & $\Delta_{D N S}$ & $1120 \times 840 \times 280$ & $\mathrm{~F}$ \\
\hline EFLES-DSM-F8-NR8 & 8 & 8 th & $8 \Delta_{D N S}$ & $\Delta_{D N S}$ & $1120 \times 840 \times 280$ & $\mathrm{~F}$ \\
\hline
\end{tabular}

TABLE 1. Abbreviations for names of simulations performed using $R e_{0}=1200$. The simulations include DNS, LES and EFLES. The listed LES and EFLES were all performed using the dynamic Smagorinsky model (DSM). In the abbreviated name, ' $C$ ' denotes a coarse grid, ' $M$ ' a medium grid, and ' $F$ ' a fine grid and each grid label is followed by a number denoting the discretization-order accuracy. $N_{R}$ represents the factor by which the drop field has been reduced in LES or EFLES compared to the DNS.

\subsection{Conventional LES performance}

The emphasis of the present work being on the two-phase flow aspect, the gas-phase results will only be succinctly discussed and differences with the compressible singlephase flow results of Radhakrishnan \& Bellan (2012a) pointed out. We note, as we did in Radhakrishnan \& Bellan (2012a), that in conventional LES the filter shape does not appear, but that it must be chosen when computing the FDNS. For this reason, we compare the LES results to both DNS and FDNS, where the FDNS was obtained by using a top-hat filter, and chose the filter width $\bar{\Delta}=\Delta x_{L E S}$ where $\Delta x_{L E S}$ is the LES grid spacing. The terminologies of 'grid' and 'mesh' are interchangeably used. If the LES is grid-spacing independent, all results should agree independently of the chosen grid spacing. Grids having $\mathrm{C}$ and $\mathrm{M}$ spacings are used in LES as shown in table 1. Unlike in EFLES, for conventional LES we do not use an F grid. This is because the relationship $\bar{\Delta}=\Delta x_{L E S}$ means that the resulting simulation would be the DNS which is already available. In all conventional LES computations, $N_{R}=8$ was used.

The assessment of the models' performance is made using the quantities listed in appendix B.

\subsubsection{The dynamic Smagorinsky model}

Similarly to the findings of Radhakrishnan \& Bellan (2012a), the Favre-averaged streamwise velocity, $\left\langle\rho u_{1}\right\rangle / \rho_{0} \Delta U_{0}$, plotted versus $\xi=x_{2} / \delta_{m}$ (not shown) is excellently 

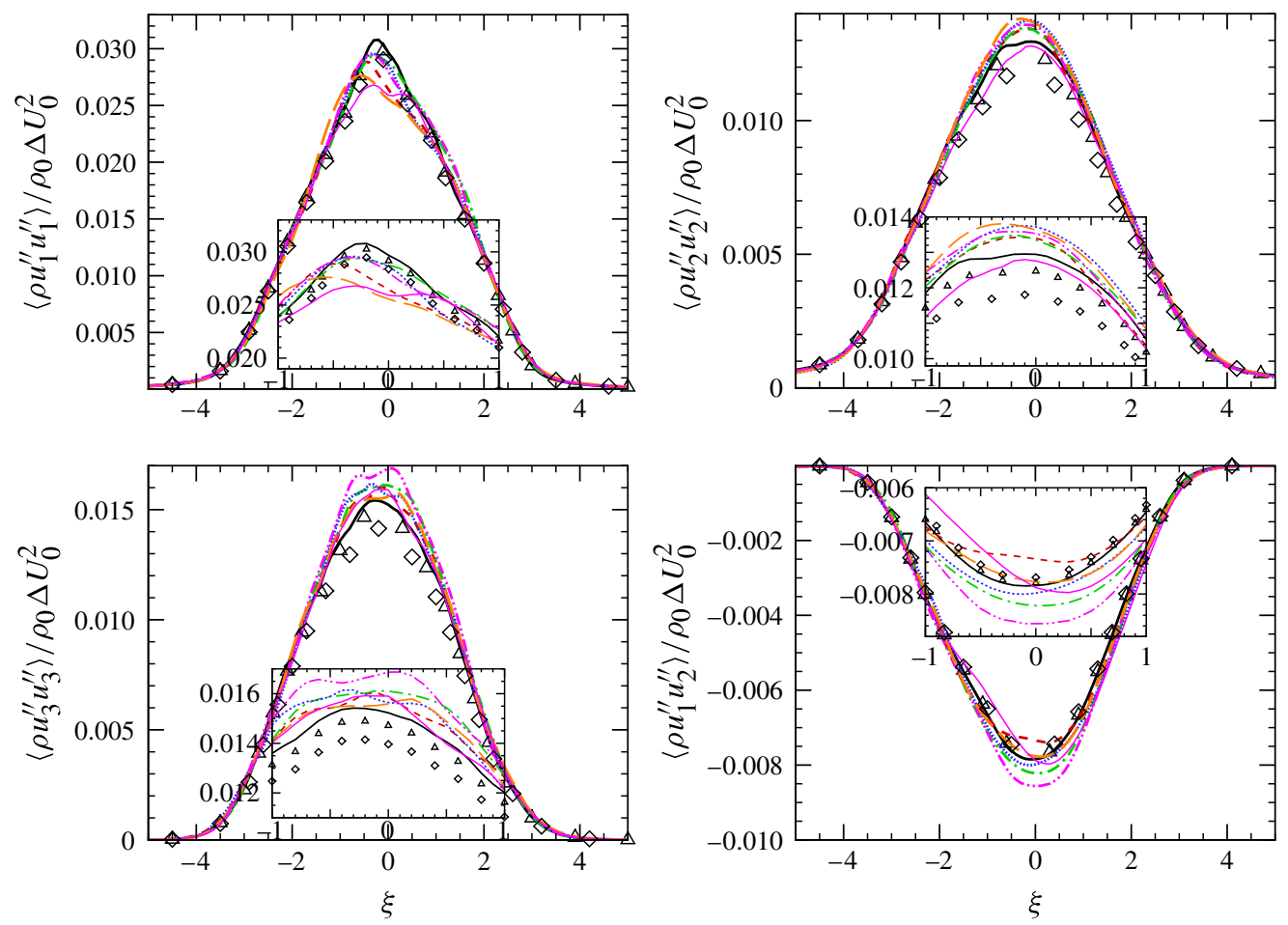

FIGURE 2. (Colour online) Reynolds stress prediction. - DNS; $\triangle$, filtered DNS with $\bar{\Delta}=2 \Delta_{D N S} ; \diamond$, filtered DNS with $\bar{\Delta}=4 \Delta_{D N S} ;--_{-}$, LES-DSM-M8-NR8; - - , LES-DSMC8-NR8; ........., LES-DSM-M6-NR8; -..-, LES-DSM-C6-NR8; _ — , LES-DSM-M4-NR8; - LES-DSM-C4-NR8.

predicted, independent of the grid spacing and discretization order, and this aspect holds for all conventional LES irrespective of the SGS model. This aspect also holds for the Favre-averaged vapour mass fraction, $\left\langle\rho Y_{v}\right\rangle / \rho_{0}$, regardless of the SGS model employed.

In concert with the findings of Radhakrishnan \& Bellan (2012a), in the central part of the layer there is a wide discrepancy among Reynolds stress predictions according to grid spacing and spatial discretization order, as shown in figure 2. For $\left\langle\rho u_{1}^{\prime \prime} u_{1}^{\prime \prime}\right\rangle / \rho_{0} \Delta U_{0}^{2}$ (quantities ()" are defined in appendix B), in contrast to the single-phase flow results, all LES predictions underestimate rather than overestimate the DNS as well as the two FDNS obtained with different filter widths. However, both $\left\langle\rho u_{2}^{\prime \prime} u_{2}^{\prime \prime}\right\rangle / \rho_{0} \Delta U_{0}^{2}$ and $\left\langle\rho u_{3}^{\prime \prime} u_{3}^{\prime \prime}\right\rangle / \rho_{0} \Delta U_{0}^{2}$ still tend to overestimate and better approximate the DNS rather than the FDNS. For $\left\langle\rho u_{1}^{\prime \prime} u_{2}^{\prime \prime}\right\rangle / \rho_{0} \Delta U_{0}^{2}$, the FDNS very closely follows the DNS (as in Radhakrishnan \& Bellan 2012a), and thus which template is better reproduced is not an issue; however, unlike in single-phase flow, the LESs do not consistently overestimate the template over the $\xi$ range.

Drop ensemble-average information, denoted by \{\} , and calculated over six instantaneous fields between $t^{*}=240$ and $t^{*}=250$ is exhibited on figure 3 . The results show excellent (for $\left\{v_{1}\right\} / \Delta U_{0}$ ) to very good (for $\left\{v_{1, r m s}\right\} / \Delta U_{0}$, $\{S t\}$ and $\left\{D^{2}\right\} /\left\{D_{0}^{2}\right\}$ ) agreement for LES. Understandably, the drop-ensemble statistics 

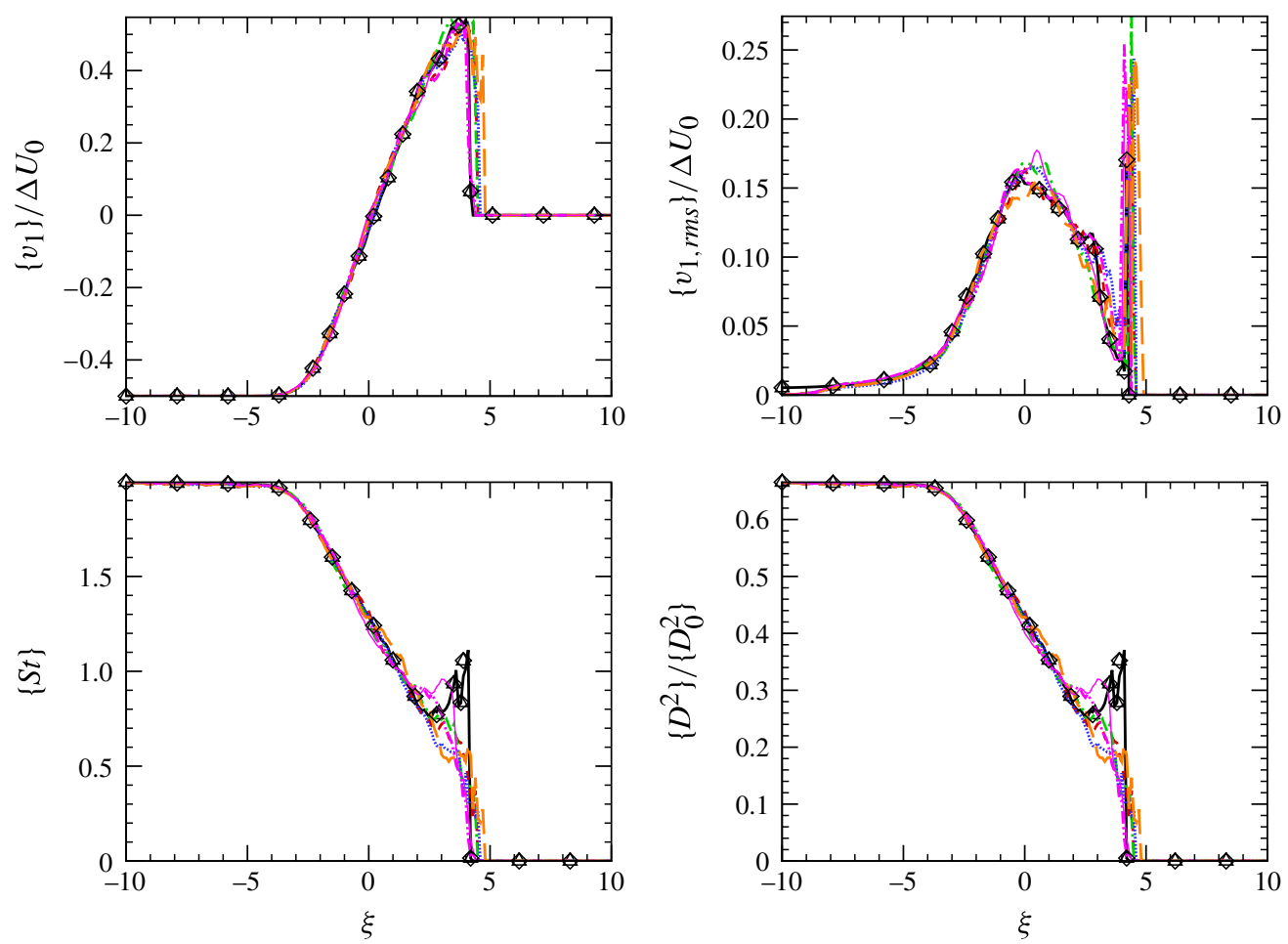

FIGURE 3. (Colour online) Drop ensemble-average statistics. - DNS; $\triangle$, filtered DNS with $\bar{\Delta}=2 \Delta_{D N S} ; \diamond$, filtered DNS with $\bar{\Delta}=4 \Delta_{D N S} ;$ - - -, LES-DSM-M8-NR8; ---, LES-DSMC8-NR8; ........., LES-DSM-M6-NR8; -..-, LES-DSM-C6-NR8; , LES-DSM-C4-NR8.

deteriorate as one moves on the $\xi$ axis from $\xi<0$ where all drops initially resided to $\xi>0$ where drops are entrained by the flow and their number is smaller.

A statistical analysis at $t^{*}=250$ over the drops located in a $\left[-4 \Delta x_{D N S},+4 \Delta x_{D N S}\right]$ slab surrounding the $x_{2}=0$ plane, with the quantity of interest conditioned over $Q$, defined by (B 6), is illustrated in figure 4(a). Compared is the total number of drops present in the slab, $N$, computed from DNS with that calculated from the FDNS and that predicted by the LES calculations. The FDNS $N$ was computed by selecting one out of 8 drops from the DNS, i.e. $N_{R}=8$, computing the statistics with the filtered DNS flow field, and then multiplying the result by $N_{R}=8$. In this manner, the FDNS is indeed the template for LES which was also computed with $N_{R}=8$. The number of drops peaks at $Q=0$, which represents the region where the magnitude of vorticity rate and strain rate are equal. The number of drops precipitously declines away from $Q=0$, and more on the $Q>0$ side corresponding to regions of high vorticity than the $Q<0$ side corresponding to regions of high strain; this effect has been experimentally observed by Fessler, Kulick \& Eaton (1994) and numerically identified in many studies (e.g. Selle \& Bellan 2007). The results show that all medium-grid LES calculations closely follow the corresponding FDNS, whereas the coarse-grid LES calculations follow the corresponding FDNS everywhere except for $Q<0$, where they underestimate $N$. It thus appears that over the $Q\left(\delta_{\omega, 0} / \Delta U_{0}\right)^{2}$ range shown in figure 4(a), predicting the number of drops at those flow locations can 

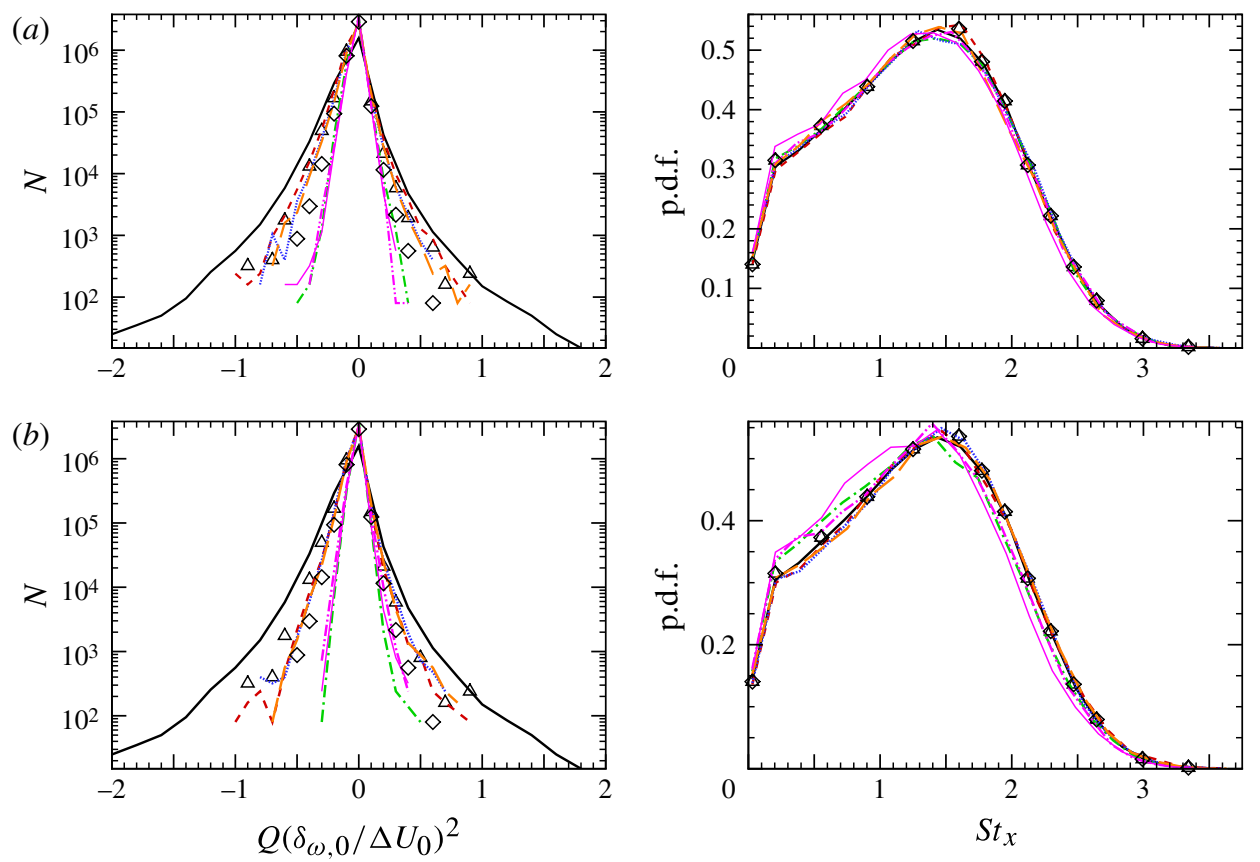

FIGURE 4. (Colour online) Drop number according to the criterion of (B 6) and Stokes number p.d.f. at $t^{*}=250$. - DNS; $\triangle$, filtered DNS with $\bar{\Delta}=2 \Delta_{D N S} ; \diamond$, filtered DNS with $\bar{\Delta}=4 \Delta_{D N S}$. $(a)$ - - -, LES-DSM-M8-NR8; -. -, LES-DSM-C8-NR8; ........, LES-DSMM6-NR8; -..-, LES-DSM-C6-NR8; _ _ , LES-DSM-M4-NR8; _ , LES-DSM-C4-NR8. (b) - - -, LES-DMM-M8-NR8; ---, LES-DMM-C8-NR8; ........, LES-DMM-M6-NR8; -..-, LES-DMM-C6-NR8; _ _ _ LES-DMM-M4-NR8; — _ LES-DMM-C4-NR8.

be effectively done rather well with a reduced, computational, drop field. The drops unaccounted for are located in regions $\left|Q\left(\delta_{\omega, 0} / \Delta U_{0}\right)^{2}\right| \gtrsim 1$, that is, in regions of smaller scales and stronger gradients unreachable by the LES calculations due to the larger mesh spacing used compared to the DNS grid spacing. When the grid is finer in LES, smaller-scale structures are better resolved, a fact which results in accurate prediction of the distribution of the drops. Okong'o \& Bellan (2004) also noted that the prediction of the drop distribution in a flow crucially depends on that of the gaseous flow since it is well known that drops tend to accumulate in regions of high strain and low vorticity.

Of note, the decline in the number of drops away from $Q=0$ means that statistics increasingly away from $Q=0$ become less reliable. This effect is found when considering $S t_{\text {ave }}$ versus $Q\left(\delta_{\omega, 0} / \Delta U_{0}\right)^{2}$ (not shown) where $S t_{\text {ave }}$ represents $S t$ computed over the drop ensemble in the above-defined slab and conditioned over $Q ; S t_{\text {ave }}<\left\{S t_{0}\right\}=3$ due to evaporation. Wide excursions are observed for $S t_{\text {ave }}$ in regions away from $Q=0$ showing that the number of drops is not conducive to trustworthy results for $S t_{\text {ave }}$ (i.e. too much variation in $S t$ for the available number of drops) except in the central region where there is a coincidence of DNS, FDNS and near coincidence with all the LES; an exception to this coincidence is $\mathrm{C} 4$ and for $0>Q\left(\delta_{\omega, 0} / \Delta U_{0}\right)^{2}>-0.2$ where generally the LES calculations behave erratically with respect to the DNS or FDNS. A more tractable measure of success for LES is the $S t$ probability density function (p.d.f.) in the $\left[-4 \Delta x_{D N S},+4 \Delta x_{D N S}\right]$ slab surrounding 

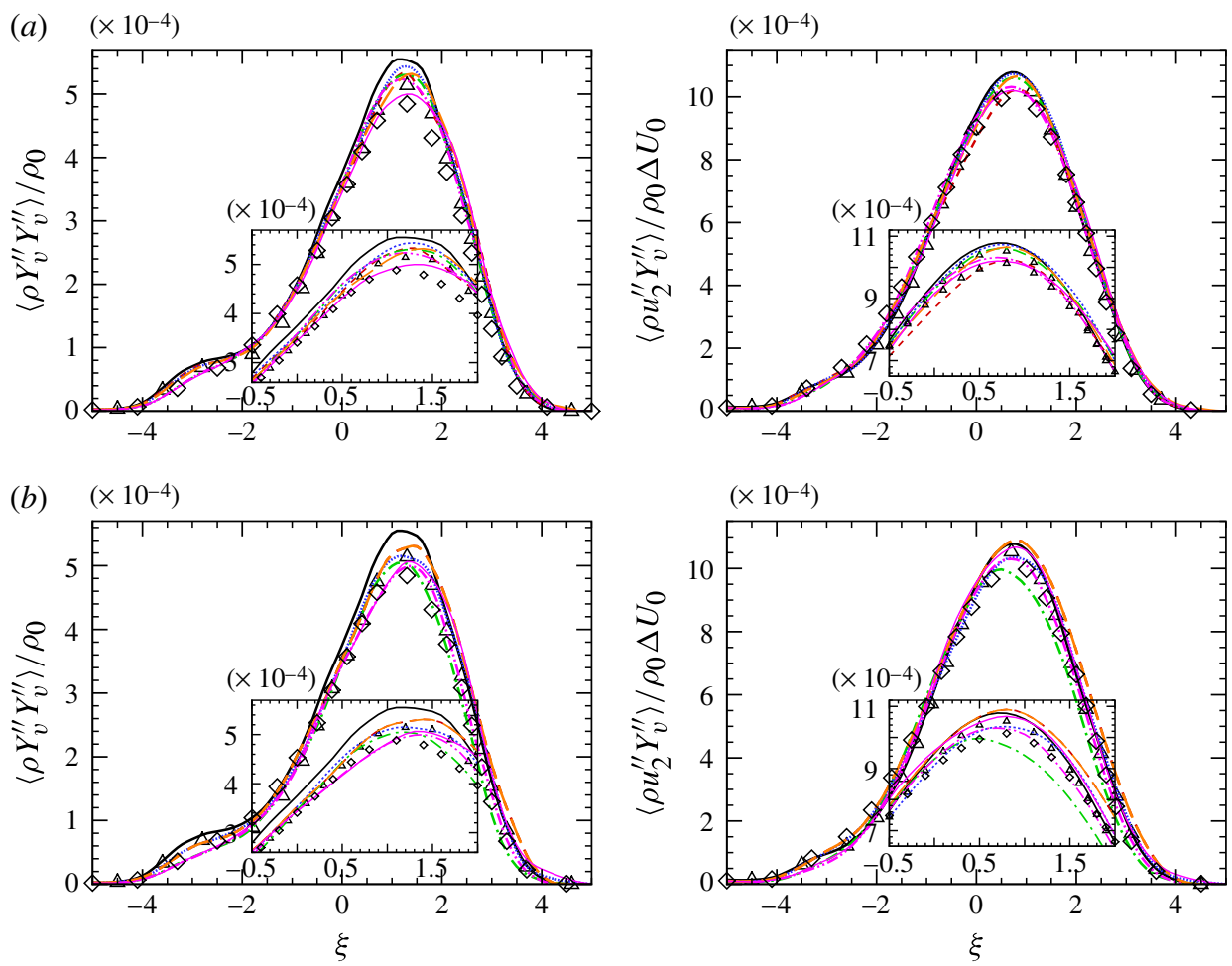

FIGURE 5. (Colour online) Vapour flux statistics from conventional LES. - DNS; $\triangle$, filtered DNS with $\bar{\Delta}=2 \Delta_{D N S} ; \diamond$, filtered DNS with $\bar{\Delta}=4 \Delta_{D N S}$. $(a)---$, LES-DSM-M8NR8; -.-, LES-DSM-C8-NR8; ........., LES-DSM-M6-NR8; -..-, LES-DSM-C6-NR8;

LES-DSM-M4-NR8; __, LES-DSM-C4-NR8. (b) _ - -, LES-DMM-M8-NR8; - - , LESDMM-C8-NR8; ........, LES-DMM-M6-NR8; -..-, LES-DMM-C6-NR8; — —, LES-DMMM4-NR8; _ _ LES-DMM-C4-NR8.

the $x_{2}=0$ plane, also shown in 4(a). It is apparent that, due to evaporation, the $S t$ p.d.f. has substantially changed from the initial condition (\$4) of a Gaussian with a peak of 3 and a standard deviation of 0.5 . The FDNS closely follows the DNS. All LES calculations with the exception of $\mathrm{C} 4$, and to a lesser extent M4, capture the p.d.f. on the smaller-than-mean drop-size side (all but for the smallest drops); however, only M4 correctly predicts the mean. On the larger-than-mean drop-size side, C4 slightly underpredicts the FDNS over the entire range, while all other LES calculations follow the FDNS rather well.

The variance of the vapour mass fraction fluctuations and the vapour flux are presented on figure $5(a)$. The profiles are skewed, indicating transport from the lower to the upper stream. Any discrepancy between LES and DNS or FDNS mainly manifests itself in the upper stream of the layer, although the C-grid LES calculations, unlike the M-grid ones, cannot capture the $\left\langle\rho Y_{v}^{\prime \prime} Y_{v}^{\prime \prime}\right\rangle / \rho_{0}$ lower stream 'bump' in the regions where evaporation is vigorous and induces substantial vapour mass fraction fluctuations. The other region where evaporation is vigorous is the lower part of the upper stream where a relatively small number of drops comes into contact with air at a temperature exceeding that of the drops. In that region, the FDNS underestimates the DNS $\left\langle\rho Y_{v}^{\prime \prime} Y_{v}^{\prime \prime}\right\rangle / \rho_{0}$; with the exception of the C4 LES which underpredicts the FDNS, 

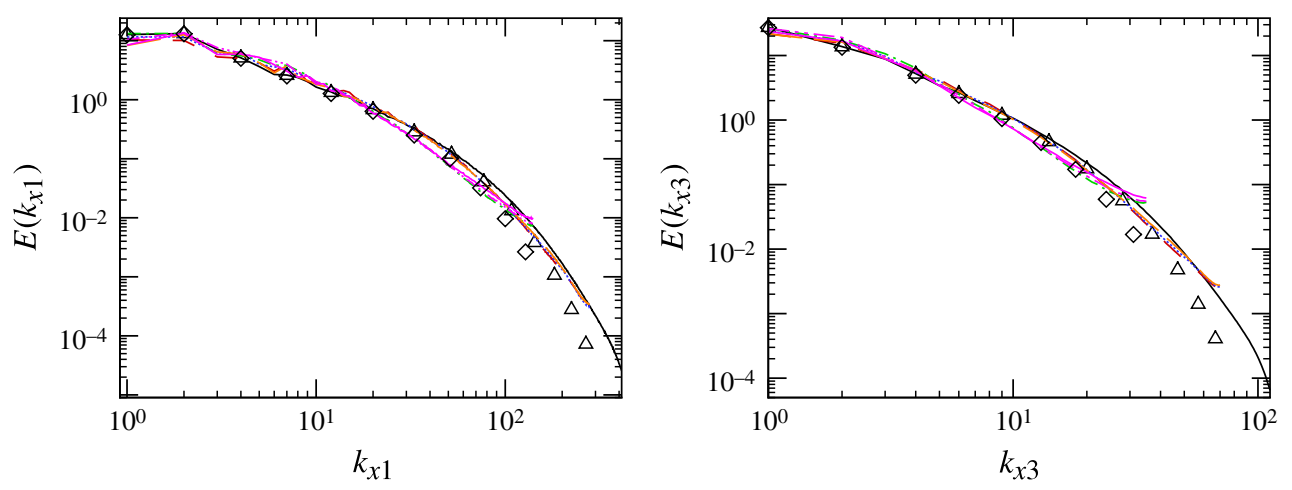

FIGURE 6. (Colour online) Turbulent kinetic energy prediction. - , DNS; $\triangle$, filtered DNS with $\bar{\Delta}=2 \Delta_{D N S} ; \diamond$, filtered DNS with $\bar{\Delta}=4 \Delta_{D N S} ;---$, LES-DSM-M8-NR8; ---, LESDSM-C8-NR8; ........, LES-DSM-M6-NR8; -..-, LES-DSM-C6-NR8; — — , LES-DSM-M4NR8; , LES-DSM-C4-NR8.

all other LES predictions are between the FDNS and DNS, with the M6 LES being closer to the DNS and the M4 LES being closest to the FDNS, without indications of improved predictions with a finer grid. Considering $\left\langle\rho u_{2}^{\prime \prime} Y_{v}^{\prime \prime}\right\rangle / \rho_{0} \Delta U_{0}$, the FDNS nearly coincides with the DNS and C6, C4 and M8 LES calculations increasingly depart from the FDNS.

Similar to the results of Radhakrishnan \& Bellan (2012a), the finding is here that all LES spectra $E\left(k_{x 1}\right)$ and $E\left(k_{x 3}\right)$ (see definition in appendix B) show overprediction at the smallest resolved scales (figure 6).

Summarizing the findings related to the Smagorinsky model, we conclude the following: the LES calculations slightly overpredict the energy at the smallest resolved scales, just as found by Radhakrishnan \& Bellan (2012a) for single-phase flow; the performance of a specified grid spacing and spatial discretization order for the gaseous flow field may not be the same in two-phase flow as for single-phase flow; none of the LES calculations is more adept than the others at predicting the drop field; and reducing the drop field by a factor of 8 does not have an impact on the prediction of the number of drops in moderate-strain or moderate-vorticity regions of the flow. Most important and surpassing the issue of accuracy is the fact that the different predictability of these LES is a manifestation of the lack of grid-spacing and discretization-order independence, both of which limit all pronouncements on accuracy.

Having analysed in detail the results for the DSM SGS model, the discussion for the other SGS models will mostly focus on: $(a)$ the Reynolds stresses to indicate any differences from the single-phase situation of Radhakrishnan \& Bellan (2012a); and (b) the vapour fluxes.

\subsubsection{The dynamic mixed model}

In figure 7 are illustrated the Reynolds stresses obtained in LES using the DMM model. In contrast to the single-phase findings, here all LES calculations underestimate $\left\langle\rho u_{1}^{\prime \prime} u_{1}^{\prime \prime}\right\rangle / \rho_{0} \Delta U_{0}^{2}$, with all M-grid LES calculations being a better approximation than the C-grid LES calculations. For $\left\langle\rho u_{2}^{\prime \prime} u_{2}^{\prime \prime}\right\rangle / \rho_{0} \Delta U_{0}^{2}$ the results emulate those of the single-phase flow in that the M-grid predictions are closer to the DNS and the C-grid results are nearer to the FDNS. Considering $\left\langle\rho u_{3}^{\prime \prime} u_{3}^{\prime \prime}\right\rangle / \rho_{0} \Delta U_{0}^{2}$, unlike in the singlephase case where the C-grid LES calculations underpredicted even the FDNS, here, all 

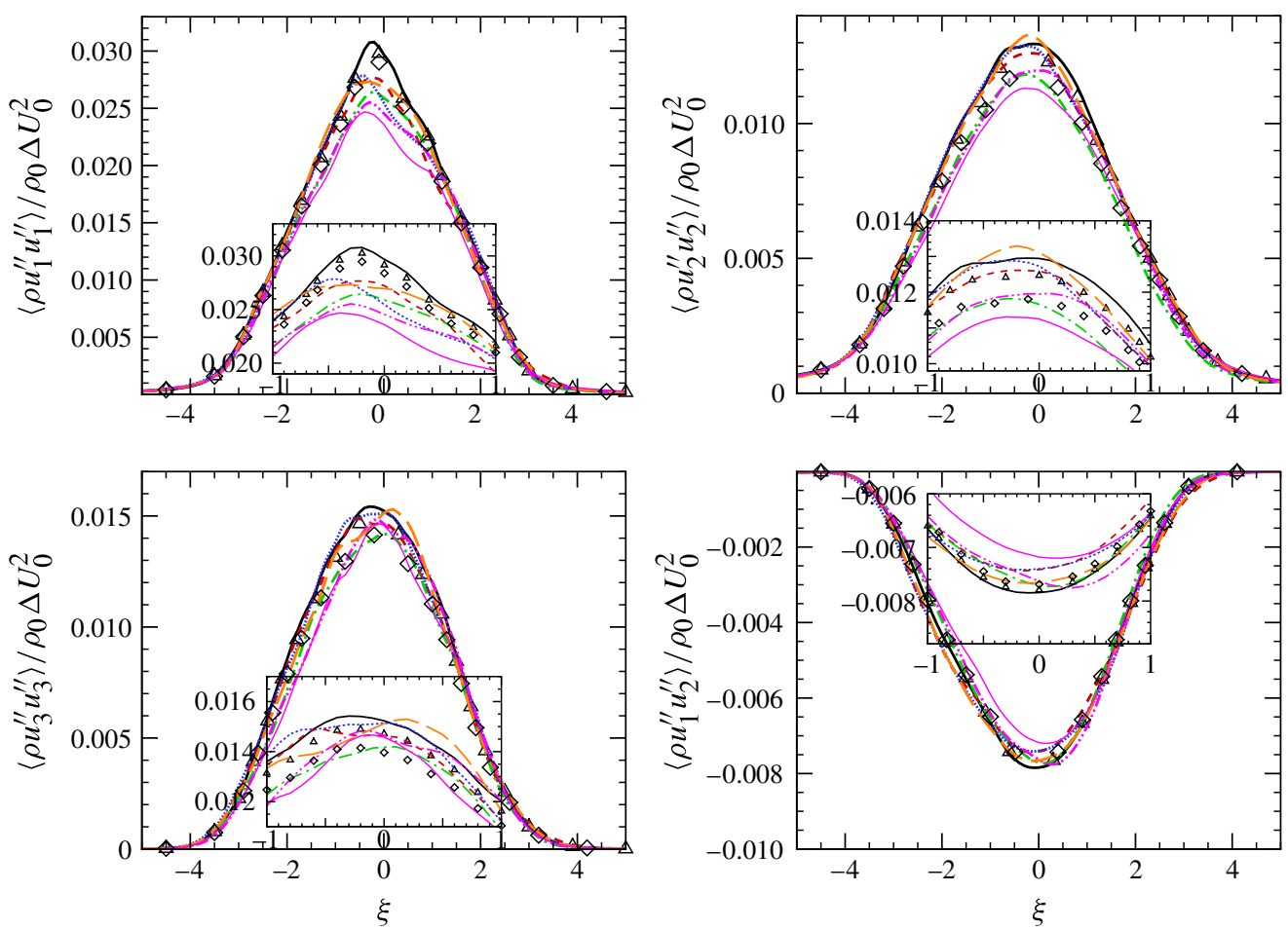

FIGURE 7. (Colour online) Reynolds stress prediction. - DNS; $\triangle$, filtered DNS with $\bar{\Delta}=2 \Delta_{D N S} ; \diamond$, filtered DNS with $\bar{\Delta}=4 \Delta_{D N S} ;$ - - -, LES-DMM-M8-NR8; --., LESDMM-C8-NR8; ........, LES-DMM-M6-NR8; -..., LES-DMM-C6-NR8; — —, LES-DMMM4-NR8; , LES-DMM-C4-NR8.

C-grid LES calculations overpredict the FDNS, with the M-grid ones being closer to the corresponding FDNS. Also dissimilar to the single-phase flow situation where the peak of $\left\langle\rho u_{1}^{\prime \prime} u_{2}^{\prime \prime}\right\rangle / \rho_{0} \Delta U_{0}^{2}$ was overpredicted, all LES calculations except M8 and M6 predict the peak value accurately. Just as for the Smagorinsky model, the conclusion is that single-phase flow results are not applicable to two-phase flow cases.

Drop conditional averaging comparisons appear on figure $4(b)$. Similar to the Smagorinsky model results, the conditionally averaged number of drops $N$ predicted by the $\mathrm{M}$ grid match the FDNS whereas the $\mathrm{C}$-grid results show underprediction near $Q<0$. The $S t$ p.d.f., also displayed in figure $4(b)$, indicates that all C-grid LES overpredict the p.d.f. for the drops smaller than the mean (except for the smallest drops), whereas all the M-grid simulations predict the FDNS results. For the larger-than-mean St, all C-grid LES underpredict the p.d.f., whereas the M-grid LES predictions correctly align with the FDNS.

Both $\left\langle\rho Y_{v}^{\prime \prime} Y_{v}^{\prime \prime}\right\rangle / \rho_{0}$ and $\left\langle\rho u_{2}^{\prime \prime} Y_{v}^{\prime \prime}\right\rangle / \rho_{0} \Delta U_{0}$ are displayed on figure 5(b). Results from the M4, C6 and C8 LES calculations for $\left\langle\rho Y_{v}^{\prime \prime} Y_{v}^{\prime \prime}\right\rangle / \rho_{0}$ all depart from the FDNS in the peak region while the other simulations flank the FDNS; the bump in the DNS and FDNS for $-4<\xi<-2$ is well captured by all M-grid simulations (although not as well as for the Smagorinsky model) but not by the C-grid ones. For $\left\langle\rho u_{2}^{\prime \prime} Y_{v}^{\prime \prime}\right\rangle / \rho_{0} \Delta U_{0}$, the LES calculations exhibit erratic behaviour in their comparison with DNS or FDNS, with the worst predictions being from $\mathrm{C} 8$ and then from $\mathrm{C} 6$, both of which 
underpredict the DNS and FDNS, while C4 only slightly overpredicts the FDNS, as does M4 to a slightly greater extent; the best results are obtained with M6 and M8.

Thus, for the DMM model there is no LES computation which consistently emerges as being the best, while, similar to the results of $\$ 6.3 .1$, the C-grid LES seem for some results to better emulate the FDNS, and the M-grid LES seem for those results to better duplicate the DNS. Similar to the LES results obtained with DSM, the different predictions of these LES show that there is neither grid-spacing independence nor discretization-order independence, and thus all aspects of LES fidelity are moot.

\subsection{Explicitly filtered LES performance}

As with single-phase flows (Radhakrishnan \& Bellan 2012a), conventional LES predictions of Reynolds stresses are both grid-spacing and spatial-discretization-order dependent. The prediction of the important two-phase flow quantities $\left\langle\rho Y_{v}^{\prime \prime} Y_{v}^{\prime \prime}\right\rangle / \rho_{0}$ or $\left\langle\rho u_{2}^{\prime \prime} Y_{v}^{\prime \prime}\right\rangle / \rho_{0} \Delta U_{0}$ is also dependent on both grid spacing and spatial discretization order employed. However, the conditionally averaged distribution of the drops $(N)$ is predicted accurately especially for $Q>0$ even with the reduced $\left(N_{R}=8\right)$ drop representation used. The $S t$ p.d.f. is best predicted by the DSM which also showed grid-spacing-independent results with $\mathrm{M}$ and $\mathrm{C}$ grids and various discretization orders.

We inquire here whether the EFLES displays grid-spacing and discretization-order independence and how this aspect affects the predictive capability compared to conventional LES. The same filter as used in the single-phase EFLES calculations of Radhakrishnan \& Bellan (2012a) is employed in the present two-phase EFLES calculations. For EFLES, $\bar{\Delta}$ is independent of the grid resolution (see table 1). Additional to the $\mathrm{C}$ and $\mathrm{M}$ grids used in conventional LES, we introduce here an $\mathrm{F}$ grid (see table 1) since EFLES conducted with the $\mathrm{F}$ grid will be different from DNS. Similar to the single-phase flow study of Radhakrishnan \& Bellan $(2012 a), \bar{\Delta}$ has a value which is 8 times the grid spacing for the F-grid calculations, 4 times the grid spacing for the M-grid calculations and 2 times the grid spacing for the $\mathrm{C}$-grid calculations. We assess here whether the results found by Radhakrishnan \& Bellan (2012a) and listed in $\S 1$ also hold for two-phase flow with evaporation. Additionally, we evaluate here how the EFLES capability depends on the drop-field SGS model by changing the value of $N_{R}$. To explore the role of explicit filtering with respect to gas-phase and drop-field SGS models, we conduct EFLES with no gas-phase SGS model using two drop-field SGS models (i.e. two values of $N_{R}$ ).

\subsubsection{The dynamic Smagorinsky model, $N_{R}=8$}

The Reynolds stresses obtained from the EFLES employing the dynamic Smagorinsky model and $N_{R}=8$ are depicted in figure 8 . Compared with the situation for conventional LES (figure 2), here the predictions of all simulations are bunched in a considerably narrower band. For all Reynolds stresses, M6 and M8 simulations produce coinciding results, with M4 showing only small deviation from the other two medium-grid simulations. However, none of the coarse-grid predictions exhibit close agreement among them or with the medium-grid results. Moreover, all EFLES calculations overestimate the magnitude of the FDNS stresses (and also those from DNS), with a single exception for $\left\langle\rho u_{1}^{\prime \prime} u_{1}^{\prime \prime}\right\rangle / \rho_{0} \Delta U_{0}^{2}$ at and in the vicinity of $\xi=0$. The turbulent kinetic energy spectra displayed in figure 9 show that all EFLES calculations match with each other and also with the FDNS at the smallest resolved scales. We seek further understanding of drop-related quantities, namely the $N$ distribution according to the $Q$ criterion of (B 6), and the $S t$ p.d.f., both of which are computed, similarly to those illustrated for the conventional LES, at $t^{* *}=250$ 

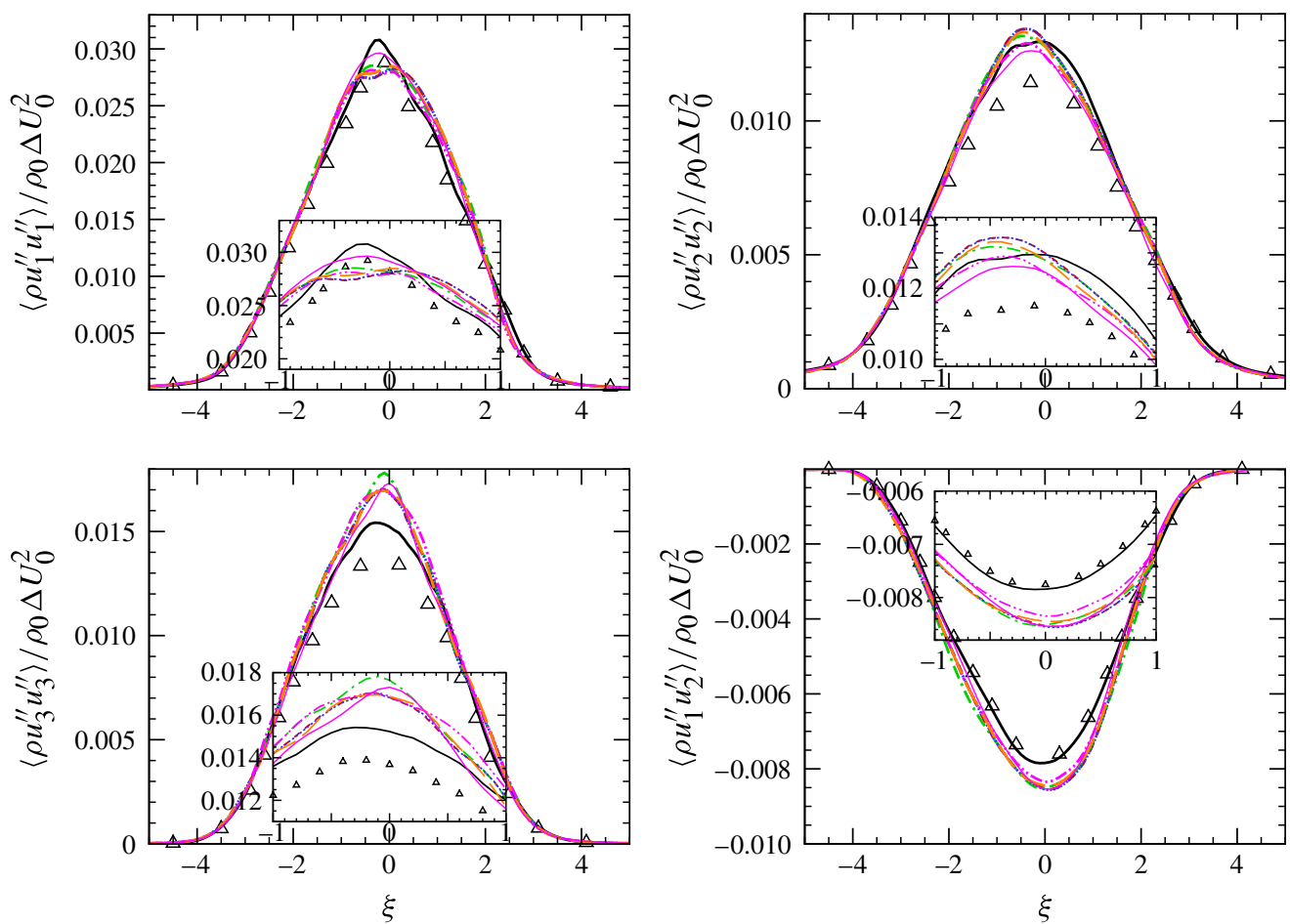

FIGURE 8. (Colour online) Reynolds stress prediction. - DNS; $\triangle$, filtered DNS; - - -, EFLES-DSM-M8-NR8; ---, EFLES-DSM-C8-NR8; ........, EFLES-DSM-M6-NR8; -..-, EFLES-DSM-C6-NR8; — — _ EFLES-DSM-M4-NR8; — , EFLES-DSM-C4-NR8.
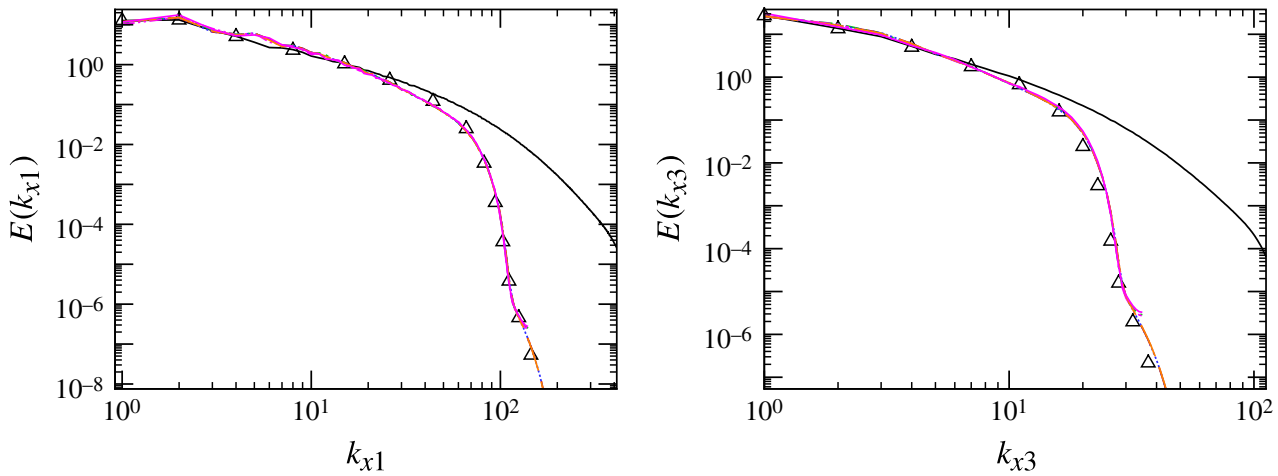

FIGURE 9. (Colour online) Turbulent kinetic energy spectra. - DNS; $\triangle$, filtered DNS; - - -, EFLES-DSM-M8-NR8; -.-,, EFLES-DSM-C8-NR8; ........, EFLES-DSM-M6-NR8; -..-, EFLES-DSM-C6-NR8; _ _ _ EFLES-DSM-M4-NR8; _ _, EFLES-DSM-C4-NR8.

in a $\left[-4 \Delta x_{D N S},+4 \Delta x_{D N S}\right]$ slab surrounding the $\left(x_{1}, x_{3}\right)$ plane at $x_{2}=0$. The results are illustrated in figure 10(a). Unlike for conventional LES, all EFLES predict an $N$ distribution similar to the FDNS. Complementary information is provided by the $S t$ p.d.f. showing that all EFLES results closely agree with the FDNS and among themselves but that the agreement is better for the $S t$ values larger than the mean; for the $S t$ values smaller than the mean but larger than $\sim 0.2$, the best agreement is given by simulations M4 and C8. 

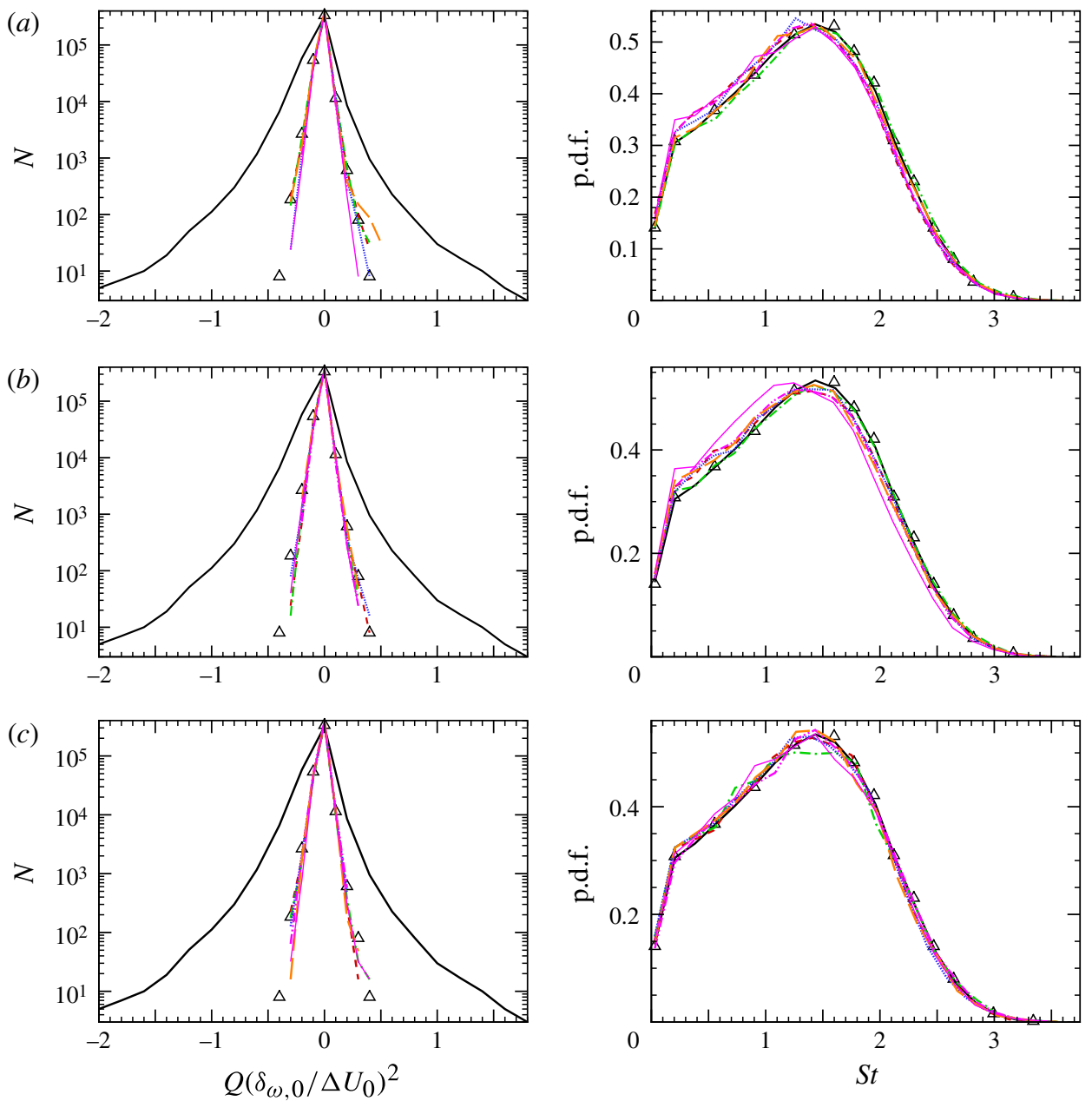

FIgURE 10. (Colour online) Drop number according to the criterion of (B 6) and Stokes number p.d.f. at $t^{*}=250$. - , DNS; $\triangle$, filtered DNS. ( $a$ ) - - -, EFLES-DSM-M8-NR8; ---, EFLES-DSM-C8-NR8; ........, EFLES-DSM-M6-NR8; -..., EFLES-DSM-C6-NR8; EFLES-DSM-M4-NR8; _ , EFLES-DSM-C4-NR8. (b) - - -, EFLES-DMM-M8-NR8; ---, EFLES-DMM-C8-NR8; ........, EFLES-DMM-M6-NR8; -..-, EFLES-DMM-C6-NR8; — — EFLES-DMM-M4-NR8; —, EFLES-DMM-C4-NR8. ( $c$ ) - - -, EFLES-DSM-M8NR16; ---, EFLES-DSM-C8-NR16; ........, EFLES-DSM-M6-NR16; -..-, EFLES-DSM-C6NR16; EFLES-DSM-M4-NR16; , EFLES-DSM-C4-NR16.

Both $\left\langle\rho Y_{v}^{\prime \prime} Y_{v}^{\prime \prime}\right\rangle / \rho_{0}$ and $\left\langle\rho u_{2}^{\prime \prime} Y_{v}^{\prime \prime}\right\rangle / \rho_{0} \Delta U_{0}$ are displayed in figure 11(a). In contrast to all conventional LES $\left\langle\rho Y_{v}^{\prime \prime} Y_{v}^{\prime \prime}\right\rangle / \rho_{0}$ predictions, here all EFLES results coincide (a minimal exception is for $\mathrm{C} 6$ in a small part of the layer), and additionally follow the FDNS, including the bump created in the lower stream by the vigorously evaporating drops. However, the agreement with the FDNS is not complete, as there is an underestimate for $0.5<\xi<3$; this evaluation of accuracy is meaningful since there is grid-spacing and discretization-order independence. The situation is somewhat more complex for $\left\langle\rho u_{2}^{\prime \prime} Y_{v}^{\prime \prime}\right\rangle / \rho_{0} \Delta U_{0}$, where although the EFLES results are globally closer to each other than their counterpart in figure 5(a), C6 and C4 produce somewhat lower 

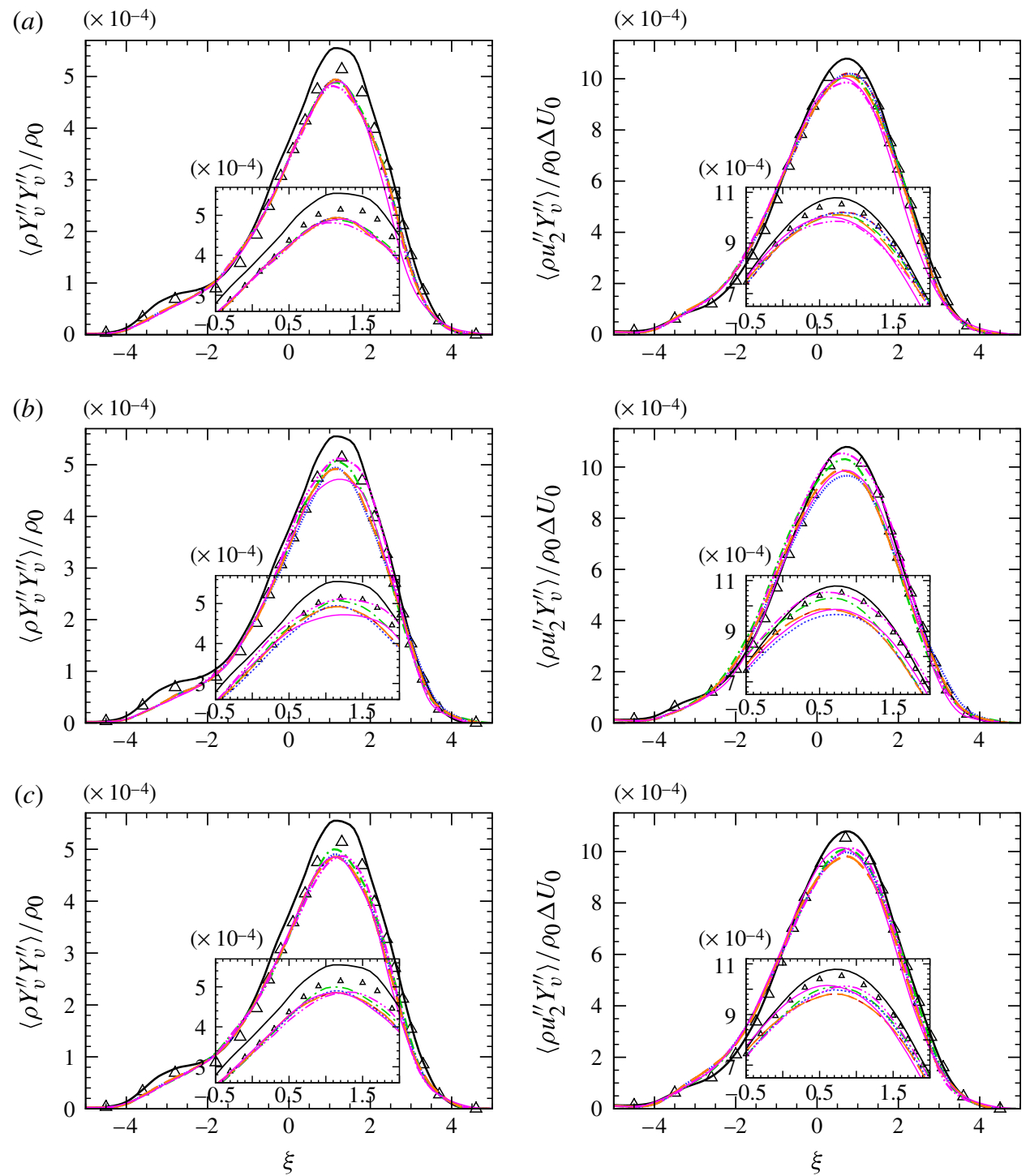

FIGURE 11. (Colour online) Vapour flux statistics from EFLES. - DNS; $\triangle$, filtered DNS. (a) - - -, EFLES-DSM-M8-NR8; - - , EFLES-DSM-C8-NR8; ........, EFLES-DSM-M6NR8; -..-, EFLES-DSM-C6-NR8; _ _ , EFLES-DSM-M4-NR8; _ , EFLES-DSM-C4NR8. $(b)$ - - -, EFLES-DMM-M8-NR8; -.-, EFLES-DMM-C8-NR8; ........, EFLES-DMMM6-NR8; -..-, EFLES-DMM-C6-NR8; _ —, EFLES-DMM-M4-NR8; — , EFLESDMM-C4-NR8. (c) - - -, EFLES-DSM-M8-NR16; -- -, EFLES-DSM-C8-NR16; EFLES-DSM-M6-NR16; -..-, EFLES-DSM-C6-NR16; _ — EFLES-DSM-M4-NR16; $\longrightarrow$, EFLES-DSM-C4-NR16.

estimates than M4 and C8, which are themselves negligibly depressed with respect to those of M8 and M6 that are the closest to the FDNS. Thus, for the M-grid spacing, all discretization-order EFLES coincide and their prediction of the FDNS is a slight underestimate. 

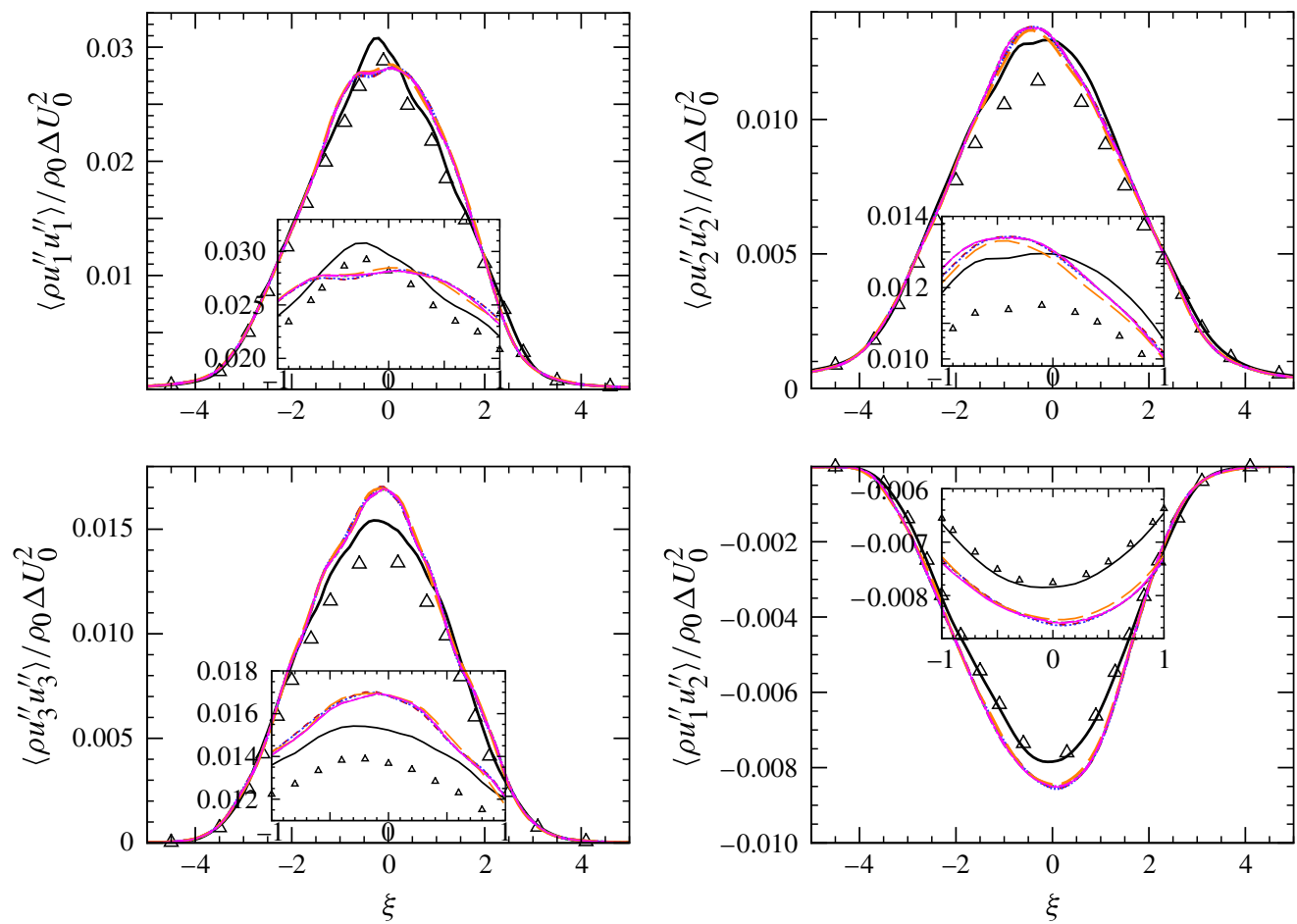

FIGURE 12. (Colour online) Reynolds stress prediction. - - DNS; $\triangle$, filtered DNS; - - -, EFLES-DSM-M8-NR8; - --, EFLES-DSM-F8-NR8; ........., EFLES-DSM-M6-NR8; -..-, EFLES-DSM-F6-NR8; — — _ EFLES-DSM-M4-NR8; —, EFLES-DSM-F4-NR8.

The present consideration of an $\mathrm{F}$ grid was motivated by the fact that unlike in the single-phase EFLES study (Radhakrishnan \& Bellan 2012a) where the results for $\mathrm{C}$ and $\mathrm{M}$ grids matched for the eighth-order scheme, the results from the $\mathrm{C}$ grid do not match for two-phase flow with those of the $\mathrm{M}$ grid even for the eighth-order scheme. Thus, to obtain an increased perspective, similar to the single-phase flow study, it was deemed necessary to also perform EFLES calculations on an $\mathrm{F}$ grid to inquire whether grid-spacing independence can be obtained by further mesh refinement. Figure 12 illustrates these results and shows that the fine-grid calculations match for all orders of discretization and additionally they coincide with the results from M6 and M8 calculations, with the M4 predictions only negligibly disagreeing. This implies that for the fourth to eighth discretization orders, the $M$ grid is sufficiently refined to represent grid-spacing-independent results since further grid refinement yields the same solution; additionally, the $\mathrm{M}$ grid is also sufficient to obtain discretization-order independence from the fourth to eighth orders. Further refinement to the $\mathrm{F}$ grid seems thus unnecessary.

Displayed in tables 2 and 3 is a comparison between the present results and those of the similar single-phase flow study (Radhakrishnan \& Bellan 2012a). Each table focuses on one aspect of this comparison. Table 2 lists the minimum filter-width to grid-spacing ratio at which grid convergence can be expected and provides the grid spacings for which results converged. Table 3 lists discretization schemes that provide the same results for a chosen grid and filter width. 


$\begin{array}{lcc} & \begin{array}{c}\text { Single-phase } \\ \left(R e_{0}=600\right)\end{array} & \begin{array}{c}\text { Two-phase } \\ \left(R e_{0}=1200\right)\end{array} \\ \text { Fourth-order discretization } & 4(\mathrm{M}, \mathrm{F}) & 4(\mathrm{M}, \mathrm{F}) \\ \text { Sixth-order discretization } & 4(\mathrm{M}, \mathrm{F}) & 4(\mathrm{M}, \mathrm{F}) \\ \text { Eighth-order discretization } & 2(\mathrm{C}, \mathrm{M}, \mathrm{F}) & 4(\mathrm{M}, \mathrm{F})\end{array}$

TABLE 2. Minimum ratio of filter width to EFLES grid spacing to achieve grid independence. Also listed are the grids for which independence has been achieved. The relationship between the EFLES and DNS grid is provided in table 1 for $\mathrm{C}, \mathrm{M}$ and $\mathrm{F}$ grids, and it is the same for single-phase and two-phase flows. The single-phase results are those of Radhakrishnan \& Bellan (2012a).

$\begin{array}{lccc} & \begin{array}{c}\text { Coarse grid } \\ (R=2)\end{array} & \begin{array}{c}\text { Medium grid } \\ (R=4)\end{array} & \begin{array}{c}\text { Fine grid } \\ (R=8)\end{array} \\ \text { Single-phase }\left(R e_{0}=600\right) & \text { N/A } & \text { 4th, 6th, 8th } & \text { 4th, 6th, 8th } \\ \text { Two-phase }\left(R e_{0}=1200\right) & \text { N/A } & \text { 4th, 6th, 8th } & \text { 4th, 6th, 8th }\end{array}$

TABLE 3. Discretization-order independence achieved for EFLES according to the grid spacing. The single-phase flow results are those of Radhakrishnan \& Bellan (2012a). $R$ is the ratio of the filter width to EFLES grid spacing. The ratio of EFLES to DNS grid spacing is listed in table 1.

Clearly, for the fourth- and sixth-order discretizations, the requirements to obtain grid-independent results are the same for two-phase flows as for single-phase flows (Radhakrishnan \& Bellan 2012a) in that the minimum filter-width to grid-spacing ratio required is 4 and the $M$ grid is that for which grid independence is achieved. However, for the eighth-order discretization, the $\mathrm{C}$ grid is that for which grid-spacing independence is obtained for single-phase flow and the minimum filter-width to grid-spacing ratio required is 2, whereas for two-phase flow the $\mathbf{M}$ grid is that for which grid independence is achieved and the minimum ratio is 4 . To elucidate this different requirement for the eighth-order scheme from single-phase flow, we invoke the different aspects of these two flows. Considering that single-phase flows with $R e_{0}=1800$ (Radhakrishnan \& Bellan 2012a) have exhibited the same behaviour as those with $R e_{0}=600$, it is apparent that the difference between the present $R e_{0}=1200$ two-phase flow and the $R e_{0}=600$ single-phase flow does not stem from the initially larger value of $R_{0}$. Rather, we attribute this difference to the presence of the drops which manifests as a source term in the vorticity equation (the term coming from $\bar{S}_{I I}$ in the momentum equation). This means that the drops promote the formation of a considerably more complex flow than a single-phase equivalent flow. As the discretization order becomes larger (i.e. 8 compared to 6 or 4), the representation of functions or derivatives becomes more accurate, meaning that more structure of the flow is retained. Although the small scales created by the nonlinear terms are filtered below the filter width, at scales above the filter width, the more complex structure of the two-phase flow obtained with the higher-order discretization will remain. However, such a structure may not be possible to solve on a $\mathrm{C}$ grid to the same extent as on $\mathrm{M}$ or $\mathrm{F}$ grids (which is a necessary requirement for grid convergence), a fact which we conjecture explains why the $\mathrm{C}$-grid results do not converge with those of the $\mathrm{M}$ and $\mathrm{F}$ grids at the specified filter width. Apparently, for the eighth-order discretization and 
the specified filter width, only the $\mathrm{M}$ grid can render this complexity as well as the F grid.

So far, we have considered grid-spacing and discretization-order solution independence when either one of these aspects was fixed, and then inquired what was the required filter-width to grid-spacing ratio necessary to obtain independence with respect to the other aspect. However, an enlarged and perhaps even more useful viewpoint can be obtained by combining the information in tables 2 and 3, each of which compares only one aspect, as stated above. For example, table 2 shows that for the present two-phase flow, grid-spacing independence is obtained for M8 for a ratio of 4 , and table 3 shows that M4 also agrees with M8 for a ratio of 4 . Therefore M4 can be considered both the grid-spacing-independent and the discretization-orderindependent solution. In the single-phase flow case, table 2 shows that $\mathrm{C} 8$ is the gridindependent solution for a minimum ratio of 2 , but because according to table $2 \mathrm{C} 8$ agrees with M8 and from table 3 M8 agrees with M4 at a ratio of 4, then C8 and M4 agree but at different filter-width to grid-spacing ratio. In other words, the minimum filter-width to grid-spacing ratio needed to obtain the grid-independent solution might be different for various discretization-order schemes, but the grid-independent solution thus obtained is also discretization-order independent. Basically, because the actual solutions obtained using various discretization-order schemes agree, this implies that the grid-independent solution is also discretization-order independent. To obtain the grid-spacing and the discretization-order independent solution, the minimum filterwidth to grid-spacing ratio listed in table 2 is required.

\subsubsection{The dynamic mixed model, $N_{R}=8$}

The EFLES computations using the DMM model provide additional perspective to the capabilities of the EFLES formulation. The Reynolds stresses depicted in figure 13 show that despite considerable improvement with respect to conventional LES regarding concurrence of simulations (figure 7), generally all C-grid simulations depart from the M-grid ones and that even the M4 simulation slightly deviates from M6 and M8, particularly for $\left\langle\rho u_{3}^{\prime \prime} u_{3}^{\prime \prime}\right\rangle / \rho_{0} \Delta U_{0}^{2}$. By comparison with conventional LES, all EFLES calculations agree better with the FDNS for $\left\langle\rho u_{1}^{\prime \prime} u_{1}^{\prime \prime}\right\rangle / \rho_{0} \Delta U_{0}^{2}$, except M8 which although it produces much better results here in most of the $\xi>-0.5$ range, it fails to improve predictions for $-1<\xi<-0.5$. Regarding $\left\langle\rho u_{2}^{\prime \prime} u_{2}^{\prime \prime}\right\rangle / \rho_{0} \Delta U_{0}^{2}$, the large spread among conventional LES predictions (figure 7) is here avoided and all EFLES calculations excluding C8, and C6 reproduce the FDNS quite well. The same observation is valid for $\left\langle\rho u_{1}^{\prime \prime} u_{2}^{\prime \prime}\right\rangle / \rho_{0} \Delta U_{0}^{2}$. Finally, although all EFLES calculations overestimate $\left\langle\rho u_{3}^{\prime \prime} u_{3}^{\prime \prime}\right\rangle / \rho_{0} \Delta U_{0}^{2}$, there is substantial improvement in the consistency of the predictions.

Drop-related quantities for the EFLES DMM model are shown in figure $10(b)$. Similar to the EFLES DSM results, but unlike the conventional LES results obtained using the DMM model, all predictions for $N$ versus $Q\left(\delta_{\omega, 0} / \Delta U_{0}\right)^{2}$ cluster on, or in the vicinity of, the FDNS. With the exception of the C4 results, all EFLES predictions of the $S t$ p.d.f. are very close for the entire p.d.f., with C8 coinciding best with the FDNS, while the curves for all $\mathrm{M}$ grids are quasi-indistinguishable.

As a last evaluation of the EFLES results with $N_{R}=8$, we focus on $\left\langle\rho Y_{v}^{\prime \prime} Y_{v}^{\prime \prime}\right\rangle / \rho_{0}$ or $\left\langle\rho u_{2}^{\prime \prime} Y_{v}^{\prime \prime}\right\rangle / \rho_{0} \Delta U_{0}$ illustrated in figure 11(b). Compared to the equivalent $\left\langle\rho Y_{v}^{\prime \prime} Y_{v}^{\prime \prime}\right\rangle / \rho_{0}$ results for conventional LES (figure $5 b$ ), here there is substantial improvement in the convergence of all $\mathrm{M}$-grid predictions, but none of the $\mathrm{C}$-grid simulations agrees with the M-grid ones; also, the M-grid predictions do not fall on the FDNS. A similar observation applies to the $\left\langle\rho u_{2}^{\prime \prime} Y_{v}^{\prime \prime}\right\rangle / \rho_{0} \Delta U_{0}$ results for which the EFLES with the 

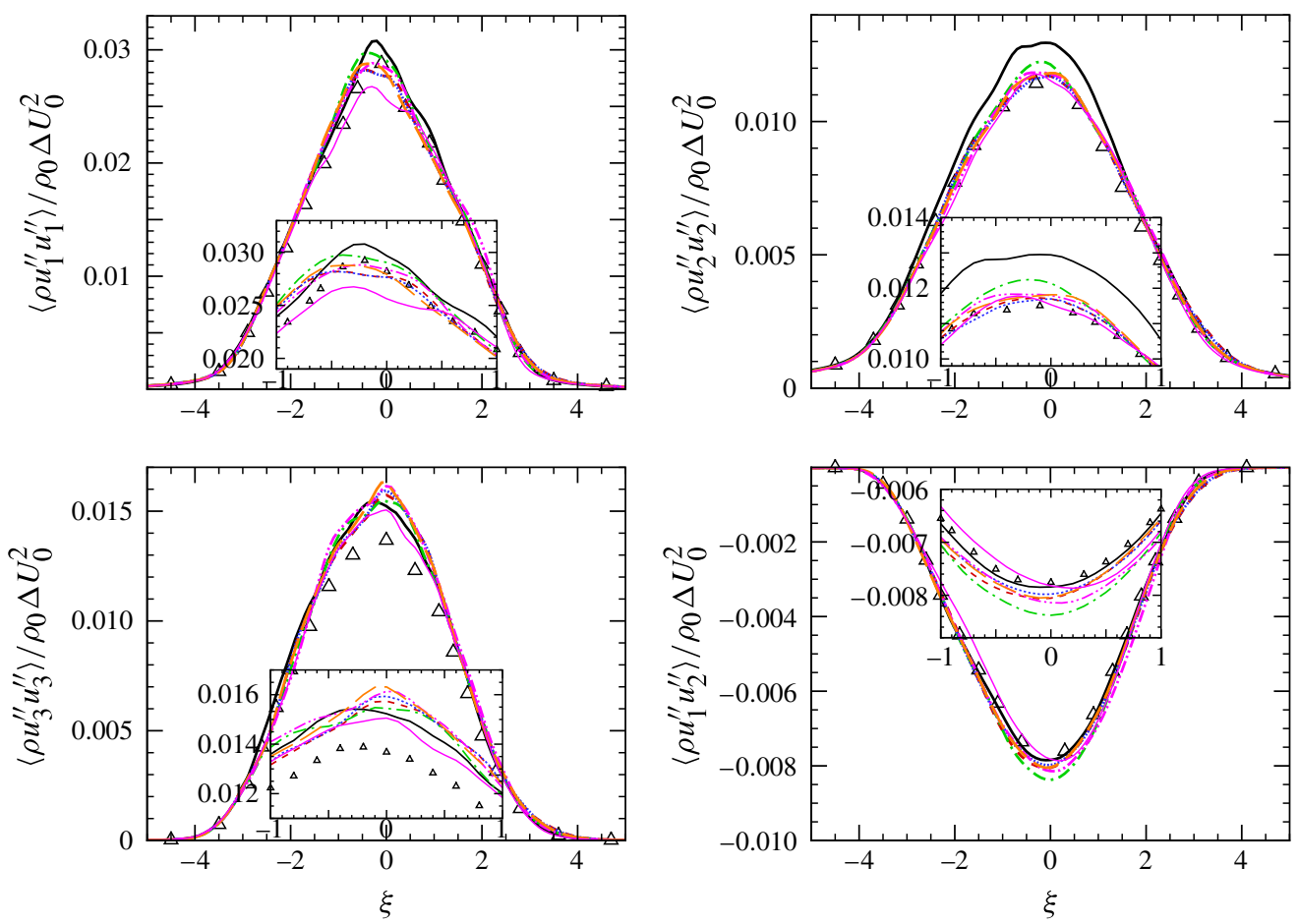

FIGURE 13. (Colour online) Reynolds stress prediction. - , DNS; $\triangle$, filtered DNS; - - -, EFLES-DMM-M8-NR8; EFLES-DMM-C8-NR8; EFLES-DMM-M6-NR8; -..-, EFLES-DMM-C6-NR8; EFLES-DMM-M4-NR8; , EFLES-DMM-C4-NR8.

$\mathrm{M}$ grids coincide, and $\mathrm{C} 4$ coincides with them; however, all of them substantially underpredict the FDNS, with the best prediction being from C6.

The present results show that for the two grid spacings and the fourth, sixth and eighth discretization orders studied, with the EFLES formulation, the predictions are more accurate with the DSM model whereas for single-phase flows the DMM model provided more accurate predictions. Generally, here the M-grid spatial-discretizationorder predictions agree indicating discretization-order independence. However, gridspacing independence cannot be established even for eighth-order discretization by comparing M- and C-grid results. Qualitatively, the trends observed for the DMM model are similar to those displayed by the DSM model. An F-grid calculation that could have proved the grid-independent nature of the $\mathrm{M}$ grid could not be performed with the DMM model because of excessive computational requirements on the computer platforms used. Since the results from the DSM EFLES are closer to grid-spacing independence and are quantitatively more accurate when compared to the FDNS, we select it rather than the DMM model EFLES to study the drop-field SGS model, that is, the $N_{R}$ (i.e. FST model) effect on the EFLES predictions.

\subsubsection{The dynamic Smagorinsky model, $N_{R}=16$}

Conducting simulations with $N_{R}=16$ rather than $N_{R}=8$ means that there are fewer drops in the field and thus potentially the vorticity production (mathematically, through the source terms in the vorticity equation) could be different. Explicit filtering removes small scales but it has no direct effect on the large flow scales. We explore here 

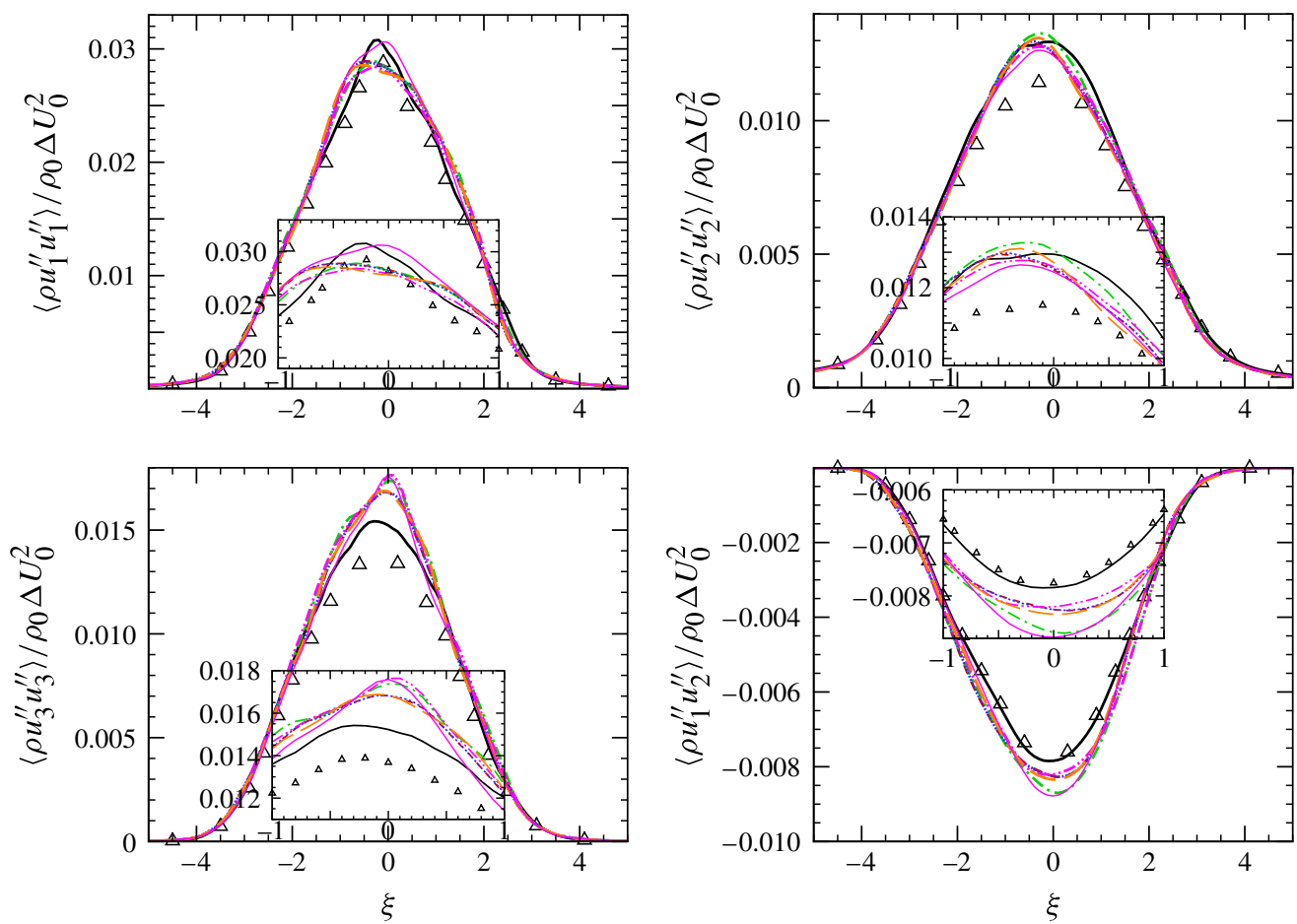

FIGURE 14. (Colour online) Reynolds stress prediction. - , DNS; $\triangle$, filtered DNS; - - -, EFLES-DSM-M8-NR16; --_, EFLES-DSM-C8-NR16; ........, EFLES-DSM-M6-NR16; -..., EFLES-DSM-C6-NR16; EFLES-DSM-M4-NR16; , EFLES-DSM-C4-NR16.

the effect that this reduction has on the results, recalling that for conventional LES Radhakrishnan \& Bellan (2012b) found substantial variation of the results with the $N_{R}$ value.

Paralleling the results obtained so far, equivalent information is provided for the Reynolds stresses in figure 14, for $N$ versus $Q\left(\delta_{\omega, 0} / \Delta U_{0}\right)^{2}$ and the $S t$ p.d.f. in figure $10(c)$, and for the vapour fluxes in figure $11(c)$. The comparison is made with the EFLES results obtained using the DSM model and $N_{R}=8$ so as to determine if and how the findings are influenced by the drop-field SGS model.

Comparison of figures 14 and 8 reveals that the results are similar for $\left\langle\rho u_{2}^{\prime \prime} u_{2}^{\prime \prime}\right\rangle / \rho_{0} \Delta U_{0}^{2}$ and $\left\langle\rho u_{3}^{\prime \prime} u_{3}^{\prime \prime}\right\rangle / \rho_{0} \Delta U_{0}^{2}$ to those for $N_{R}=8$ in terms of convergence of predictions among simulations, but somewhat inferior for $\left\langle\rho u_{1}^{\prime \prime} u_{1}^{\prime \prime}\right\rangle / \rho_{0} \Delta U_{0}^{2}$ and $\left\langle\rho u_{1}^{\prime \prime} u_{2}^{\prime \prime}\right\rangle / \rho_{0} \Delta U_{0}^{2}$. However, considering comparisons of the EFLES results with $N_{R}=16$ and conventional LES results with $N_{R}=8$ (figures 14 and 2), it is clear that the EFLES predictions of these latter quantities agree much better among them over the two grid spacings and three discretization orders than those obtained with the conventional LES.

Examining figures $10(a)$ and $10(c)$, the conclusion is that for $N$ versus $Q\left(\delta_{\omega, 0} / \Delta U_{0}\right)^{2}$ there is a slightly better convergence of the predictions from simulations with two different grid spacings when $N_{R}=16$, but that at the mean of the St p.d.f., the convergence of the six simulations is not as good as when $N_{R}=8$; however, for $S t$ smaller than the mean, the agreement among the six simulation is better when $N_{R}=16$. 

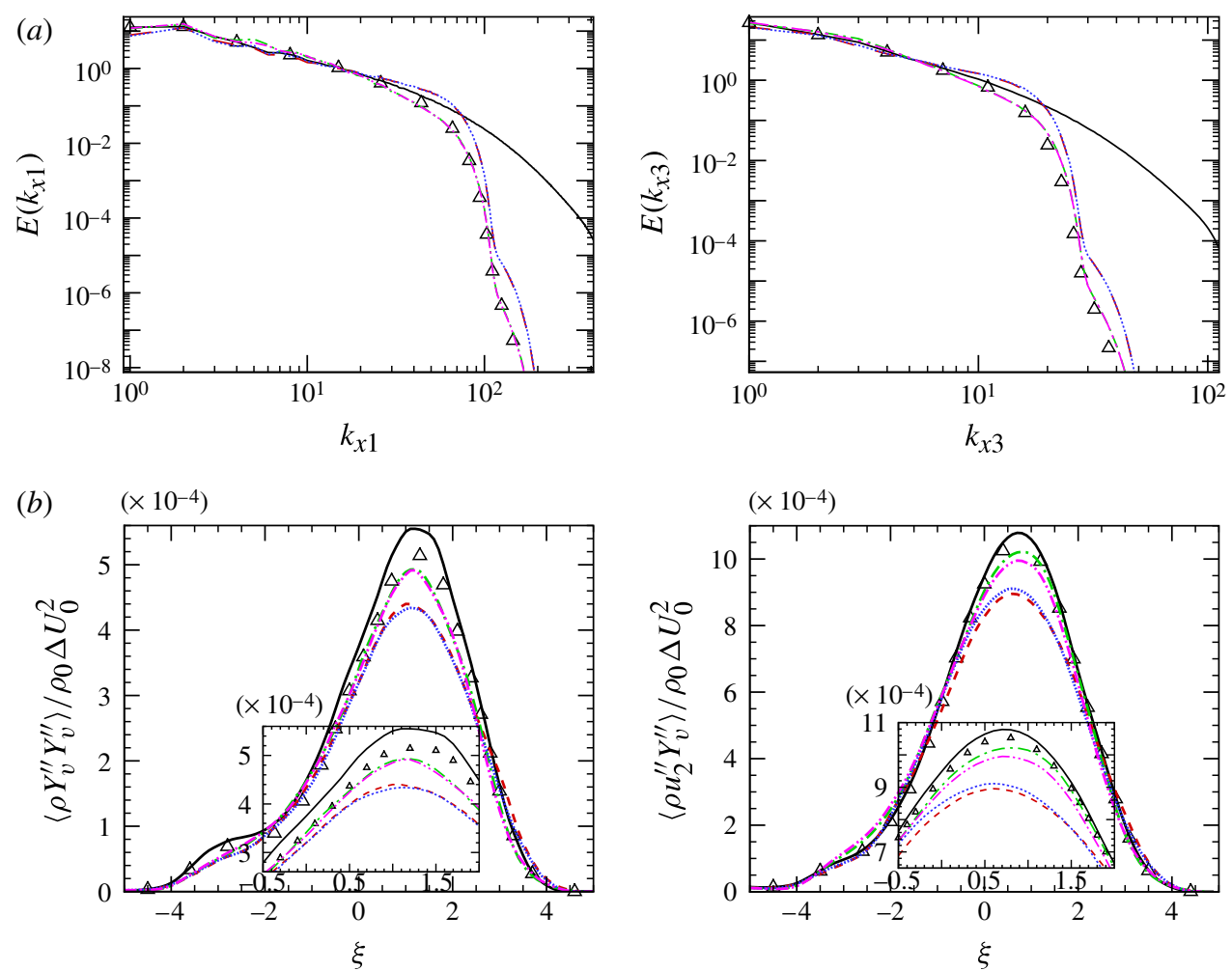

FIGURE 15. (Colour online) (a) Turbulent kinetic energy spectra, (b) vapour flux. - , DNS; $\triangle$, filtered DNS; - - , EFLES-NM-M8-NR8; --,, EFLES-DSM-M8-NR8; ........, EFLESNM-M8-NR16; -..-, EFLES-DSM-M8-NR16.

Finally, the influence of the $N_{R}$ value on $\left\langle\rho Y_{v}^{\prime \prime} Y_{v}^{\prime \prime}\right\rangle / \rho_{0}$ or $\left\langle\rho u_{2}^{\prime \prime} Y_{v}^{\prime \prime}\right\rangle / \rho_{0} \Delta U_{0}$ can be assessed by scrutinizing figures $11(a)$ and $11(c)$. For both quantities, only small differences can be observed, leading to the conclusion that the feature of converging results among simulations is practically independent of $N_{R}$ in this range of $N_{R}$ variation.

\subsubsection{Effect of explicit filtering compared to gas-phase SGS modelling}

Since both explicit filtering and gas-phase SGS modelling operate at scales smaller than the filter width, it is important to differentiate between their effects and inquire whether explicit filtering alone could provide sufficient dissipation, thus rendering moot the use of a gas-phase SGS model. A similar inquiry was performed for singlephase compressible flow by Radhakrishnan \& Bellan (2012a) and it was clear that gas-phase SGS models were necessary in EFLES to achieve the demonstrated success. However, because in two-phase flows the small scales have an additional origin than in single-phase flow, and because the drop-field SGS model also plays a role, the conclusion from Radhakrishnan \& Bellan (2012a) may not necessarily hold.

Illustrated in figure 15 is a comparison of the spectra (figure 15a) and the vapour fluxes $\left\langle\rho Y_{v}^{\prime \prime} Y_{v}^{\prime \prime}\right\rangle / \rho_{0}$ or $\left\langle\rho u_{2}^{\prime \prime} Y_{v}^{\prime \prime}\right\rangle / \rho_{0} \Delta U_{0}$ (figure 15b) from calculations using either the DSM model or no gas-phase SGS model ('no-model' labelled NM) for two drop-field SGS models: $N_{R}=8$ and $N_{R}=16$. All simulations were conducted using M8 which displayed grid-spacing and discretization-order independence when using DSM EFLES 
(see $§ 6.4 .1$ ). The results for the spectra show, independent of the value of $N_{R}$, an accumulation of energy for the NM cases whereas no such accumulation is seen for the DSM EFLES. The vapour fluxes show that the results are independent of the $N_{R}$ value but that they substantially differ between the DSM and NM cases. Since the gas-phase SGS model assumes an increasingly important role with increasing Reynolds number, the comparison would be even more unfavourable for the larger $R e$ values which are of interest for practical applications. The conclusion is that the EF methodology indeed allows the separation of numerical and modelling errors.

\section{Summary and conclusions}

The explicitly filtered large-eddy simulation (EFLES) formulation which has been previously developed and examined for compressible single-phase flows, has been here formulated and assessed for two-phase flows with phase change. Similar to the singlephase EFLES formulation, the small-scale-producing nonlinear terms in the governing equations are explicitly filtered, and this procedure is applied both to the differential equations and to the equation of state. We examined whether the single-phase results can be replicated in the two-phase case. First, we created a template database of direct numerical simulation (DNS) which portrays the evolution of an initially perturbed three-dimensional shear layer laden with evaporating droplets; this template has served as the basis for comparison determining the accuracy of both conventional LES and EFLES.

Both conventional LES and EFLES computations have been conducted with several subgrid-scale (SGS) models. Here, the SGS models are of two generic types: for the gas phase and for the drop field. Because the definition of the SGS terms in the gas conservation equations is different in EFLES from the conventional LES, only some of the typically used SGS models could be used in EFLES computations. A single drop-field SGS model was used for conventional LES but two such models were used for EFLES, one being the model used for conventional LES. For conventional LES, the filter width was chosen to be equal to the grid spacing. For EFLES, the filter width was independent of the grid spacing. Results were obtained at three different spatial discretization orders - fourth order, sixth order and eighth order - and with either a coarse or medium grid for conventional LES, and with a coarse, medium or fine grid for EFLES. Examination of these results revealed that: (a) Unlike for conventional LES where the results are always grid dependent, the EFLES results are gridindependent for sufficiently large filter-width to grid-spacing ratio. The EFLES results were independent of the drop-field SGS models used in the range examined here. $(b)$ The filter-width to grid-spacing ratio required to obtain grid-independent results is the same for the two-phase case compared to the single-phase case for the two lower-order discretizations, but larger by a factor of two for the eighth-order discretization. In other words, the coarse grid does not exhibit grid-spacing independence for the eighth-order discretization in contrast to the single-phase flow results. This different requirement was explained by the increased complexity of two-phase flows, even at scales larger than the filter, compared to single-phase flows. (c) An enlarged interpretation of the results leads to the conclusion that although the minimum filter-width to gridspacing ratio necessary to obtain the grid-independent solution might be different for various discretization-order schemes, the grid-independent solution thus obtained is also discretization-order independent. 


\section{Acknowledgements}

This work was conducted at the Jet Propulsion Laboratory, California Institute of Technology and sponsored by the National Aeronautics and Space Administration (NASA) under the Fundamental Aeronautics Program, Subsonic Wing Program from NASA Glenn Research Center with Drs D. Bulzan and N.-S. Liu serving as program monitors and by the NASA Exploration Systems Mission Directorate/Advanced Capabilities Division under the LASER program. The computational resources were provided by the JPL Supercomputing Center and by the NASA AMES Supercomputing Center.

\section{Appendix A. Model coefficient calculation}

The dynamic model of Germano et al. (1991) is employed to calculate the coefficients in the SGS models, based on the temporal and spatial state of the flow. Because in the Germano et al. (1991) model the turbulent stresses at grid and test filter levels are related by an identity, a fact which produces an overdetermined set of equations for calculating the SGS-model coefficients, we solve this overdetermination problem using the least-square minimization proposed by Lilly (1992). Specifically, we follow Martin, Piomelli \& Candler (2000) to compute the coefficients for a compressible flow both because the Mach number is larger than the generally accepted value of 0.3 separating the incompressible and compressible regimes and because the source terms in the governing equations enhance the compressible character of the flow. The model of Martin et al. (2000) has been developed for conventional LES and is briefly described in $\S$ A.1. The adaptation of this model for EFLES is presented in $\S$ A.2.

\section{A.1. Conventional LES model coefficient calculation}

The resolved turbulent stress at test filter level can be calculated as

$$
L_{i j}=\left(\frac{\widehat{\overline{\rho u_{i} \rho u_{j}}}}{\bar{\rho}}\right)-\left(\frac{\widehat{\rho u_{i} \rho u_{j}}}{\widehat{\bar{\rho}}}\right),
$$

where $\left({ }^{\wedge}\right)$ refers the filtering at test filter width which, as generally recommended, has twice the width of the grid filter.

For the Smagorinsky model, the Germano et al. (1991) identity relates $L_{i j}$ to modelled stresses at the grid and test filter level as

$$
L_{i j}=C_{S M}^{2} \beta_{i j}-\widehat{C_{S M}^{2} \alpha_{i j}},
$$

where

$$
\begin{aligned}
& \alpha_{i j}=-2 \bar{\Delta}^{2} \bar{\rho}|S(\bar{\phi})|\left[S_{i j}(\bar{\phi})-\frac{\delta_{i j}}{3} S_{k k}(\bar{\phi})\right], \\
& \beta_{i j}=-2 \widehat{\Delta}^{2} \widehat{\bar{\rho}}|S(\widehat{\bar{\phi}})|\left[S_{i j}(\widehat{\bar{\phi}})-\frac{\delta_{i j}}{3} S_{k k}(\widehat{\bar{\phi}})\right] .
\end{aligned}
$$

As explained above, (A 2) represents five equations for one unknown coefficient, and the proposal of Lilly (1992) leads to

$$
\begin{aligned}
C_{S M}^{2} & =\frac{\left\langle L_{i j} M_{i j}\right\rangle}{\left\langle M_{k l} M_{k l}\right\rangle}, \\
M_{i j} & =\beta_{i j}-\widehat{\alpha_{i j}},
\end{aligned}
$$


where \langle\rangle refers the averaging along the homogeneous planes which for the present shear layer is $\left(x_{1}-x_{3}\right)$. Similarly,

$$
C_{Y O}=\frac{\left\langle L_{k k}\right\rangle}{\left\langle P_{m} P_{m}\right\rangle}
$$

where

$$
\begin{gathered}
P_{m}=\beta-\widehat{\alpha} \\
\alpha=\bar{\Delta}^{2} S^{2}(\bar{\phi}), \quad \beta=\widehat{\Delta}^{2} S^{2}(\widehat{\bar{\phi}}) .
\end{gathered}
$$

Finally, $\operatorname{Pr}_{\text {sgs }}$ is calculated as

$$
P r_{s g s}=\frac{C_{S M}^{2}\left\langle T_{k} T_{k}\right\rangle}{\left\langle K_{j} T_{j}\right\rangle}
$$

where

$$
\begin{gathered}
T_{j}=\theta_{j}-\widehat{\psi}_{j}, \\
\theta_{j}=-\widehat{\Delta}^{2} \widehat{\bar{\rho}}|S(\widehat{\bar{\phi}})| \frac{\partial h(\widehat{\bar{\phi}})}{\partial x_{j}}, \quad \psi_{j}=\bar{\Delta}^{2} \bar{\rho}|S(\bar{\phi})| \frac{\partial h(\bar{\phi})}{\partial x_{j}}, \\
K_{j}=\left(\frac{\widehat{\overline{\rho u_{j}} \overline{\rho h}}}{\bar{\rho}}\right)-\left(\frac{\widehat{\rho u_{j}} \widehat{\overline{\rho h}}}{\widehat{\bar{\rho}}}\right) .
\end{gathered}
$$

For the dynamic mixed model, $C_{S M}$ is calculated as

$$
C_{S M}^{2}=\frac{\left\langle L_{i j} M_{i j}\right\rangle-\left\langle N_{i j} M_{i j}\right\rangle}{\left\langle M_{k l} M_{k l}\right\rangle}, \quad C_{Y O}=\frac{\left\langle L_{k k}-N_{k k}\right\rangle}{\left\langle P_{m} P_{m}\right\rangle},
$$

with

$$
\begin{aligned}
& N_{i j}=B_{i j}-\widehat{A_{i j}},
\end{aligned}
$$

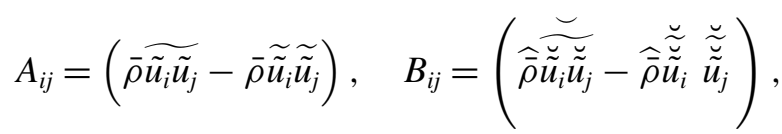

where generically

$$
\breve{\varphi}=\widehat{\bar{\rho} \varphi} / \widehat{\hat{\rho}}
$$

Similarly, $P r_{s g s}$ is calculated as

$$
\operatorname{Pr}_{s g s}=\frac{C_{S M}^{2}\left\langle T_{k} T_{k}\right\rangle}{\left\langle K_{j} T_{j}\right\rangle-\left\langle V_{j} T_{j}\right\rangle},
$$

where

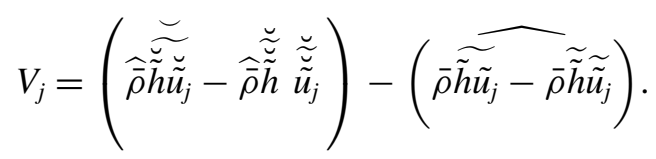




\section{A.2. Explicitly filtered LES model coefficient calculation}

The EFLES dynamic coefficient calculation follows a parallel methodology, but now with new definitions for the relevant quantities. For example, the resolved turbulent stress at test filter level can be calculated as

$$
L_{i j}^{e f}=\left(\frac{\widehat{\overline{\rho u_{i} \rho u_{j}}}}{\bar{\rho}}\right)-\left(\frac{\widehat{\widehat{\rho u_{i} \rho u_{j}}}}{\widehat{\bar{\rho}}}\right),
$$

and the equivalent definitions to quantities computed in $\S$ A.1 are

$$
\begin{aligned}
& M_{i j}^{e f}=\widehat{\beta_{i j}}-\widehat{\widehat{\alpha_{i j}}}, \\
& P_{m}^{e f}=\widehat{\beta}-\widehat{\bar{\alpha}}, \\
& K_{j}^{e f}=\left(\overline{\frac{\overline{\rho u_{j} \rho h}}{\bar{\rho}}}\right)-\left(\frac{\widehat{\widehat{\rho u_{j}} \widehat{\widehat{\rho h}}}}{\widehat{\bar{\rho}}}\right) \text {, } \\
& A_{i j}^{e f}=\left(\bar{\rho} \widetilde{\tilde{u}_{i} \tilde{u}_{j}}-\overline{\bar{\rho}} \widetilde{\tilde{u}_{i}} \widetilde{\tilde{u}}_{j}\right) \text {, }
\end{aligned}
$$

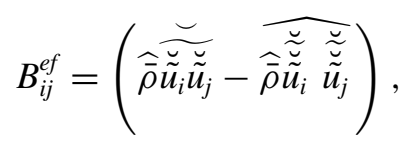

and

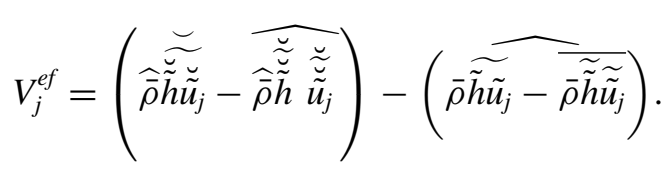

\section{Appendix B. Quantities for evaluation of LES and EFLES performance}

The performance of various models will be evaluated according to several quantities, for which we deem it important to have LES results match those provided by the DNS template. Borrowing the terminology used by Geurts \& Frohlich (2002), Meyers, Geurts \& Baelmans $(2003,2005)$ and Meyers, Sagaut \& Geurts (2006), we call these quantities 'objectives' to indicate that indeed these may be the objectives of a person undertaking LES.

(a) Zeroth- (i.e. integral) and first-order quantities, such as $\delta_{m}(t)$ and mean flow variables, all of which we consider the minimum requirement to match in any simulation. Reynolds-averaged quantities are obtained by $\left(x_{1}, x_{3}\right)$ plane averaging, and thus they are denoted by \langle\rangle . Favre-averaged quantities are denoted by \langle\rangle$^{F}$ obtained from filtered quantities, so that

$$
\langle\psi\rangle_{L E S}=\langle\bar{\psi}\rangle, \quad\langle\psi\rangle_{L E S}^{F}=\frac{\langle\overline{\rho \psi}\rangle}{\langle\bar{\rho}\rangle} .
$$

(b) Second-order quantities such as Reynolds stresses, vapour mass fraction variance, turbulent vapour fluxes and spectra. Reynolds fluctuations and Favre fluctuations are calculated as $\psi^{\prime}=\psi-\langle\psi\rangle_{L E S}$ and $\psi^{\prime \prime}=\psi-\langle\psi\rangle_{L E S}^{F}$. Reynolds stresses $R_{i j}$ are defined as

$$
R_{i j}=\frac{\left\langle\bar{\rho} u_{i}^{\prime \prime} u_{j}^{\prime \prime}\right\rangle}{\rho_{0} \Delta U_{0}^{2}} .
$$


The vapour mass fraction variance and the turbulent vapour flux are defined as

$$
(V A R)_{y v}=\frac{\left\langle\rho Y_{v}^{\prime \prime} Y_{v}^{\prime \prime}\right\rangle}{\rho_{0}}, \quad(V F L)_{i}=\frac{\left\langle\rho u_{i}^{\prime \prime} Y_{v}^{\prime \prime}\right\rangle}{\rho_{0} \Delta U_{0}},
$$

respectively.

Streamwise spectra of turbulent kinetic energy are computed as

$$
E\left(k_{1}\right)=\frac{1}{2}\left(u_{1}\left(k_{1}\right) u_{1}^{*}\left(k_{1}\right)+u_{2}\left(k_{1}\right) u_{2}^{*}\left(k_{1}\right)+u_{3}\left(k_{1}\right) u_{3}^{*}\left(k_{1}\right)\right),
$$

where $u_{1}\left(k_{1}\right), u_{2}\left(k_{1}\right)$ and $u_{3}\left(k_{1}\right)$ are the Fourier transforms taken along the $x_{1}$ direction of velocity fluctuations $u_{1}^{\prime}, u_{2}^{\prime}$ and $u_{3}^{\prime}, k$ labels the wavenumber and the superscript ()$^{*}$ denotes the complex conjugate. Spanwise spectra of turbulent kinetic energy are computed from

$$
E\left(k_{3}\right)=\frac{1}{2}\left(u_{1}\left(k_{3}\right) u_{1}^{*}\left(k_{3}\right)+u_{2}\left(k_{3}\right) u_{2}^{*}\left(k_{3}\right)+u_{3}\left(k_{3}\right) u_{3}^{*}\left(k_{3}\right)\right),
$$

where $u_{1}\left(k_{3}\right), u_{2}\left(k_{3}\right)$ and $u_{3}\left(k_{3}\right)$ are the Fourier transforms taken along the $x_{3}$ direction of velocity fluctuations $u_{1}^{\prime}, u_{2}^{\prime}$ and $u_{3}^{\prime}$.

(c) Conditional statistics of drops with respect to the difference between vorticity magnitude and strain-rate magnitude

$$
Q=-\frac{1}{2}\left(\frac{\partial u_{i}}{\partial x_{j}} \frac{\partial u_{j}}{\partial x_{i}}\right)=-\frac{1}{2}\left(S_{i j} S_{i j}-\Omega_{i j} \Omega_{i j}\right),
$$

where $\Omega_{i j}$ is the rotation-rate tensor and $S_{i j}$ is the strain-rate tensor. In regions where the vorticity dominates over the strain rate, $Q$ is positive and in regions where the strain rate dominates over the vorticity, $Q$ is negative.

\section{REFERENCES}

Bardina, J., Ferziger, J. \& Reynolds, W. 1980 Improved subgrid scale models for large eddy simulation. AIAA Paper 80-1357.

Bini, M. \& Jones, W. P. 2008 Large-eddy simulation of particle-laden flows. J. Fluid Mech. 614, 207-252.

Boivin, M., Simonin, O. \& SQuires, K. 1998 Direct numerical simulation of turbulence modulation by particles in isotropic turbulence. J. Fluid Mech. 375, 235-263.

Carati, D, Winckelmans, G. S. \& Jeanmart, H. 2001 On the modelling of the subgrid-scale and filtered-scale stress tensors in large-eddy simulation. J. Fluid Mech. 442, 119-138.

Clark, R., Ferziger, J. \& Reynolds, W. 1979 Evaluation of subgrid-scale models using an accurately simulated turbulent flow. J. Fluid Mech. 91 (1), 1-16.

Crowe, C., Chung, J. \& Troutt, T. 1998 Particle mixing in free shear flows. Prog. Energy Combust. Sci. 14, 171-194.

Fessler, J. R., Kulick, J. D. \& Eaton, J. K. 1994 Preferential concentration of heavy particles in a turbulent channel flow. Phys. Fluids 6 (11), 3742-3749.

Germano, M., Piomelli, U., Moin, P. \& Cabot, W. 1991 A dynamic subgrid-scale eddy viscosity model. Phys. Fluids A 3 (7), 1760-1765.

Geurts, B. J. \& Frohlich, J 2002 A framework for predicting accuracy limitations in large-eddy simulation. Phys. Fluids 14 (6), L41-L44.

Gore, R. A. \& Crowe, C. 1989 Effect of particle size on modulating turbulence intensity. Intl J. Multiphase Flow 286, 229-255.

Hardalupas, Y., Taylor, A. \& Whitelaw, J. 1989 Velocity and particle-flux characteristics of turbulent particle-laden jets. Proc. R. Soc. Lond. A 426, 31-78.

IsRaeli, M. \& ORSZAG, S. A. 1981 Approximation of radiation boundary conditions. J. Comput. Phys. 41, 115-135. 
Kennedy, C. \& Carpenter, M. 1994 Several new numerical methods for compressible shear layer simulations. Appl. Numer. Maths 14, 397-433.

Kennedy, C. A. \& Gruber, A. 2008 Reducing aliasing formulations of the convective terms within the Navier-Stokes equations for a compressible fluid. J. Comput. Phys. 227, 1676-1700.

Leboissetier, A., Okong'o, N. \& Bellan, J. 2005 Consistent large-eddy simulation of a temporal mixing layer laden with evaporating drops. Part 2. A posteriori modelling. J. Fluid Mech. 523, 37-78.

LESIEUR, M. 1997 Turbulence in Fluids. Kluwer.

LILLY, D. 1992 A proposed modification of the Germano subgrid-scale closure method. Phys. Fluids A 4 (3), 633-635.

LiU, S., Meneveau, C. \& Katz, J. 1994 On the properties of similarity subgrid-scale models as deduced from measurements in a turbulent jet. J. Fluid Mech. 275, 83-119.

Martin, M. P., Piomelli, U. \& CAndler, G. V. 2000 Subgrid-scale models for compressible Large-Eddy Simulation. Theor. Comput. Fluid Dyn. 13, 361-376.

Mattsson, K. \& Nordstrom, J. 2004 Summation by parts operators for finite difference approximations of second derivatives. J. Comput. Phys. 199, 503-540.

Meyers, J., Geurts, B. J. \& Baelmans, M. 2003 Database analysis of errors in large-eddy simulation. Phys. Fluids 15 (9), 2740-2755.

Meyers, J., Geurts, B. J. \& Baelmans, M. 2005 Optimality of the dynamic procedure for large-eddy simulations. Phys. Fluids 17, 0451108-1-9.

Meyers, J., Sagaut, P. \& Geurts, B. J. 2006 Optimal model parameters for multi-objective large-eddy simulations. Phys. Fluids 18, 095103-1-12.

Miller, R. \& Bellan, J. 1999 Direct numerical simulation of a confined three-dimensional gas mixing layer with one evaporating hydrocarbon-droplet laden stream. J. Fluid Mech. 384, 293-338.

OKONG'O, N. \& Bellan, J. 2004 Consistent large eddy simulation of a temporal mixing layer laden with evaporating drops. Part 1 . Direct numerical simulation, formulation and a priori analysis. J. Fluid Mech. 499, 1-47.

PAntano, C. \& SARKAR, S. 2002 A study of compressibility effects in the high-speed, turbulent shear layer using direct simulation. J. Fluid Mech. 451, 329-371.

Poinsot, T. \& LELE, S. 1992 Boundary conditions for direct simulations of compressible viscous flows. J. Comput. Phys. 101, 104-129.

Pope, S. B. 2000 Turbulent Flows. Cambridge University Press.

Pope, S. B. 2004 Ten questions concerning the large-eddy simulation of turbulent flows. New J. Phys. 6, 35-59.

Pruett, C., Sochacki, J. \& Adams, N. 2001 On Taylor-series expansions of residual stress. Phys. Fluids 13 (9), 2578-2589.

RADHAKRISHNAN, S. \& Bellan, J. 2012a Explicit filtering to obtain grid-spacing independent and discretization-order independent large eddy simulation of compressible single-phase flow. J. Fluid Mech. 697, 399-435.

RADHAKRISHNAN, S. \& BELLAN, J. $2012 b$ Influence of computational drop representation in LES of a mixing layer with evaporating drops. Comput. Fluids 58, 15-26.

Selle, L. C. \& Bellan, J. 2007 Characteristics of transitional multicomponent gaseous and drop-laden mixing layers from direct numerical simulation: composition effects. Phys. Fluids 19 (6)063301-1-33.

SMAGORINSKY, J. 1963 General circulation experiments with the primitive equations: part 1, basic experiments. Mon. Weath. Rev. 91, 99-164.

SMAGORINSKY, J. 1993 Some historical remarks on the use of nonlinear viscosities. In Large Eddy Simulation of Complex Engineering and Geophysical Flows (ed. B. Galperin \& S. Orszag), (ch. 1), pp. 3-36. Cambridge University Press.

Stolz, S. \& AdAms, N. A. 1999 An approximate deconvolution procedure for large-eddy simulation. Phys. Fluids 11 (7), 1699-1701. 
Stolz, S., AdAms, N. A. \& Kleiser, L. 2001 An approximate deconvolution model for large-eddy simulation with application to incompressible wall-bounded flows. Phys. Fluids $\mathbf{1 3}$ (4), 997-1015.

Vanella, M., Piomelli, U. \& Balaras, E. 2008 Effect of grid discontinuities on large-eddy simulation statistics and flow fields. J. Turbul. 9 (32), 1-23.

Williams, F. 1965 Combustion Theory. Addison-Wesley.

YoshizawA, A. 1986 Statistical theory for compressible turbulent shear flows, with the application to subgrid modelling. Phys. Fluids 29 (7), 2152-2164. 